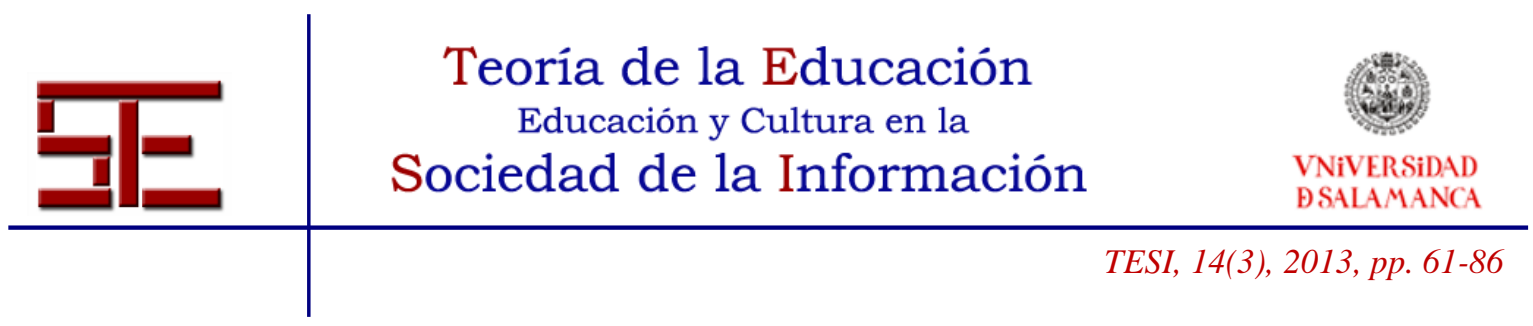

\title{
SOCIEDAD DEL CONOCIMIENTO Y ENTORNO DIGITAL
}

Resumen: La existencia y extensión del entorno digital, ha hecho posible la difusión generalizada de los instrumentos informáticos mediante los que el acceso a la información se ha ampliado como nunca en la historia de la humanidad.

A pesar de haberse analizado las consecuencias de este fenómeno y señalarse los posibles efectos no deseados en lo que se han denominado 'brechas digitales' originadas por las diferencias de acceso a la sociedad de la información por razones socioeconómicas, poco se ha explorado lo que el entorno digital supone para la construcción del conocimiento y el desarrollo de la sociedad del conocimiento.

El entorno digital es un instrumento muy potente en el acceso a la información pero, por si mismo, no incrementa las posibilidades de construcción de conocimiento y desarrollo humano, lo que puede señalar un nuevo riesgo de 'brecha digital': la vinculada a la diferencia de capacidades entre los individuos que dominan competencias y estrategias básicas necesarias para construir conocimiento, y los que sólo son usuarios pasivos de las informaciones a que se accede a través del entorno digital.

El presente trabajo explora cuestiones asociadas a diferencias individuales en las capacidades necesarias para utilizar las ventajas del entorno digital en el acceso y construcción de conocimiento válido, y defiende que el dominio de conocimientos previos, mediatiza los procesos construcción de conocimientos a partir de las informaciones a que accede cada individuo en el entorno digital, determinando su papel como individuo capacitado para la participación en el desarrollo de la sociedad del conocimiento.

Palabras clave: Dominio de conocimientos; entorno digital; tratamiento de la información; representación de conocimiento; construcción social del conocimiento






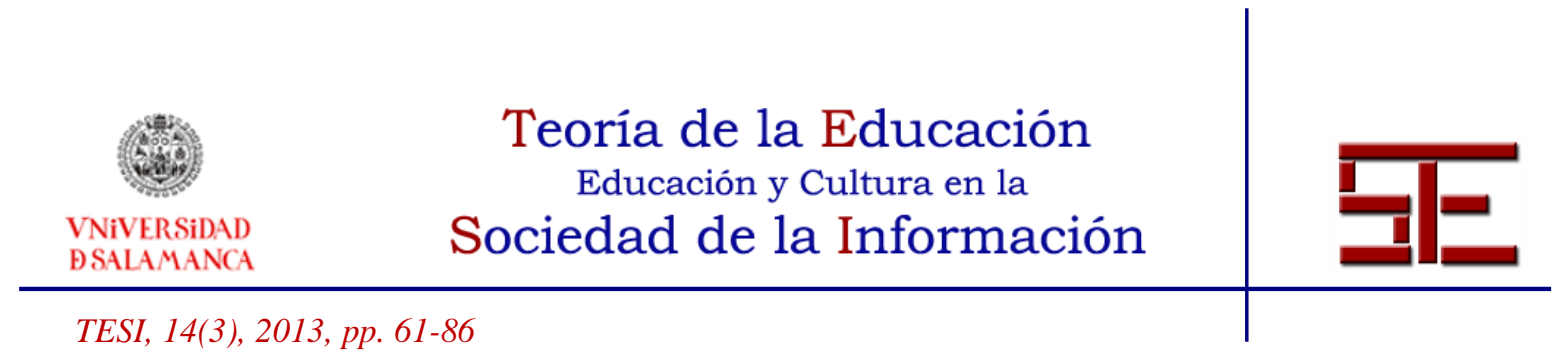

\section{KNOWLEDGE SOCIETY AND DIGITAL ENVIRONMENT}

Abstract: The existence and extension of the digital environment has made possible the broad distribution of computer equipment, allowing greater access to information than ever before in human history.

Although the consequences of this phenomenon have been analysed and the possible undesirable effects of 'digital divides' caused by different levels of access to the information society due to socio-economic reasons identified, there has been little study of what the digital environment supposes for the construction of knowledge and the development of the knowledge society.

Although the digital environment is a powerful means of accessing information, it does not necessarily increase the possibilities of constructing knowledge and human development. This may lead to a new risk of the 'digital divide', tied to the growing inequality in the abilities of those who have mastered the basic capabilities and strategies needed to build knowledge, and those who are merely passive users of the information that can be accessed through the digital environment.

This work explores issues related to individual differences in the capabilities needed to use the digital environment to access and construct valid knowledge, and defends that prior domain knowledge, mediate the processes involved in building knowledge from the information that all individuals access through the digital environment, defining their social role as an individual capable of participating in the development of the knowledge society.

Key words: Domain knowledge; digital environment; information processing; knowledge representation; social construction of knowledge.






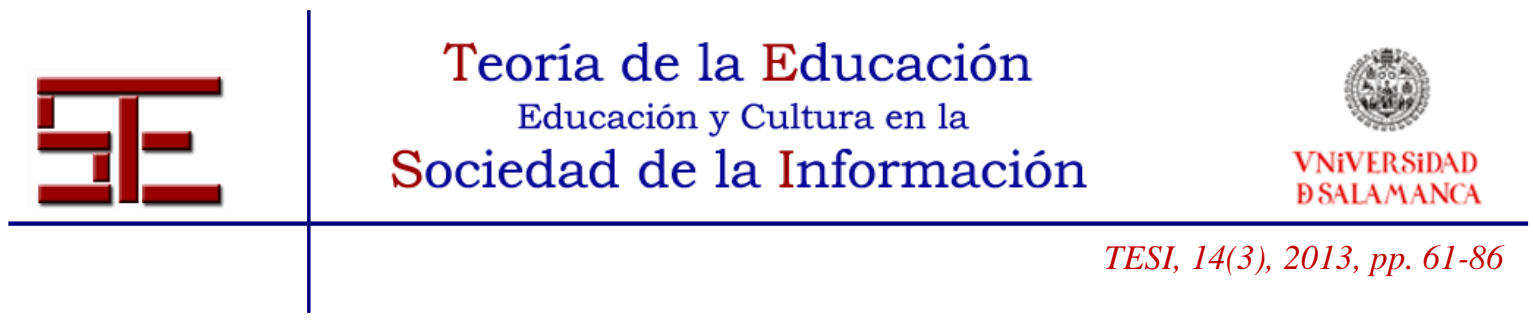

\section{SOCIEDAD DEL CONOCIMIENTO Y ENTORNO DIGITAL}

Fecha de recepción: 07/05/2013; fecha de aceptación: 10/07/2013; fecha de publicación: 30/11/2013

Clara Barroso Jerez

cbarroso@ull.es

Universidad de La Laguna

\section{INTRODUCCIÓN}

El conocimiento humano es una propiedad emergente que supera la cantidad y característica de las informaciones que procesa cada individuo. Para construir conocimiento el individuo ha de poseer competencias para dotar de interpretación a las informaciones y convertirlas en información significativa. En este proceso, la percepción del contexto en que se producen o adquieren informaciones juega una función necesaria para establecer el grado de validez de los posibles significados atribuibles a cada información. En términos individuales, esto va a constituir lo que denominaremos su dominio de conocimientos.

En términos sociales, toda sociedad se constituye como un conjunto de individuos que comparten básicamente tres ámbitos: el gnoseológico, el ético y el ámbito técnico o tecnológico.

El ámbito gnoseológico está constituido por todo aquello que configura el conocimiento de la realidad compartida por dicha sociedad. Así podemos reconocer como componentes de este ámbito lo que la sociedad sabe sobre el entorno físico en que se desarrolla la vida social (biosfera, geosfera, etc.). Las actividades que esa sociedad reconoce como propias y adecuadas para intervenir y relacionarse con el entorno físico, así como los modos de relación entre los diversos individuos que componen esa sociedad constituyen la sociosfera. Los actos y artefactos que hacen posible intervenir en el entorno para el logro de los objetivos deseables por la sociedad, constituirá la tecnosfera (Commoner, 1992). Por último, el ámbito ético de la sociedad está compuesto por todos aquellos componentes que determinan los valores y objetivos que comparten los individuos de dicha sociedad. Éstos establecerán cómo la sociedad se desarrolla en interacción con el entorno en que habita; cómo interaccionan los individuos en dicha sociedad, y cómo van a participar en las decisiones y actuaciones que se lleven a cabo; el ámbito ético estará también involucrado en cómo y para qué se utilizarán las técnicas y tecnologías de que la sociedad dispone.

En estos términos toda sociedad debe ser, por su propia naturaleza, una sociedad de conocimiento compartido. Se puede caracterizar como aquella en la que los procesos de desarrollo individual y social se asientan sobre la construcción compartida de significa-

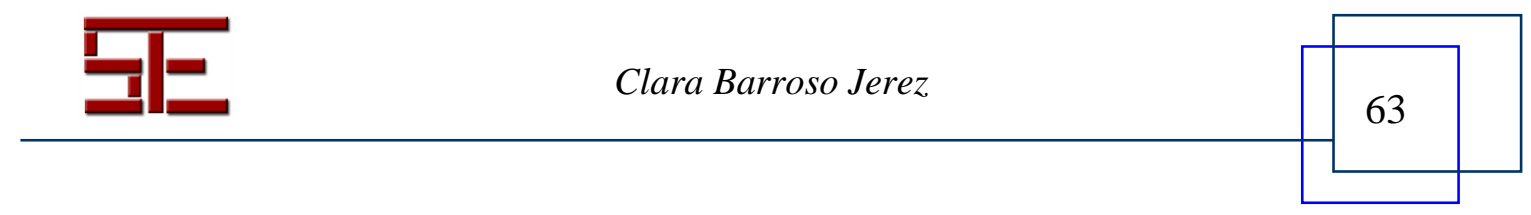






dos en interacción con el mundo físico, tecnológico y social en que los individuos se desenvuelven (Bruner, 2001). El conocimiento se construye a través de la experiencia compartida en la que cada individuo evoluciona en interacción con el entorno sociocultural en que se desenvuelve (Dewey, 2004). El conjunto de habilidades y competencias que cada individuo esté en disposición de utilizar para comprender las informaciones que utiliza y participar en el impulso y desarrollo de los componentes culturales de su sociedad, constituye su dominio social de conocimiento; en éste se asentará su capacidad para ser un miembro activo en la sociedad del conocimiento.

La sociedad de la información, vinculada a la sociedad del conocimiento, sería aquella que hace de la transmisión de informaciones el soporte para la amplificación y desarrollo de los conocimientos, valores y tecnologías o técnicas disponibles. Actualmente, la sociedad de la información está vinculada necesariamente a las tecnologías que hacen posible dicha transmisión: las tecnologías de la información (TIC). Su desarrollo ha dado lugar a lo que conocemos como 'entorno digital' que constituye un nuevo ámbito de 'realidad' compartida en dicha sociedad. De acuerdo con lo anteriormente afirmado, la pertenencia de cada individuo a esa sociedad de la información y el conocimiento, exigirá que pueda estar en disposición de reconocerse y actuar en el entorno digital para participar en el desarrollo de la misma compartiendo conocimientos y valores, incrementando así su capacidad de desarrollo personal y social. Este desiderátum, como veremos más adelante, es meramente eso, una aspiración que, en las actuales circunstancias, no se vincula al fomento de las competencias necesarias para impulsar la existencia de ciudadanos con facultades imprescindibles para ser miembros activos en la sociedad del conocimiento.

En el presenta artículo se distingue entre sociedad del conocimiento (aquella en la que todos los miembros de la sociedad poseen capacidades y competencias para ser miembros activos en la construcción social del conocimiento) y la sociedad de la información (vinculada a las posibilidades de difusión de información que ofrece el entorno digital). Se exploran los fundamentos de la construcción y dominio de conocimiento diferenciándolos de los procesos de uso y acceso a la información; se distinguen capacidades (en términos de posibilidades y habilidades de uso de hardware y software) y competencias (en términos de utilización del entorno digital en procesos de construcción del conocimiento). Se aborda un análisis de las características del entorno digital, las posibilidades que ofrece en la difusión de informaciones y los límites en la construcción del conocimiento, defendiendo que la construcción del conocimiento es más que la capacidad o habilidad vinculada a la alfabetización digital relacionada con el uso de hardware y software.

En este trabajo se defiende que lo que hace posible que la sociedad de la información sea un instrumento que impulse la sociedad del conocimiento, se vincula a competen-

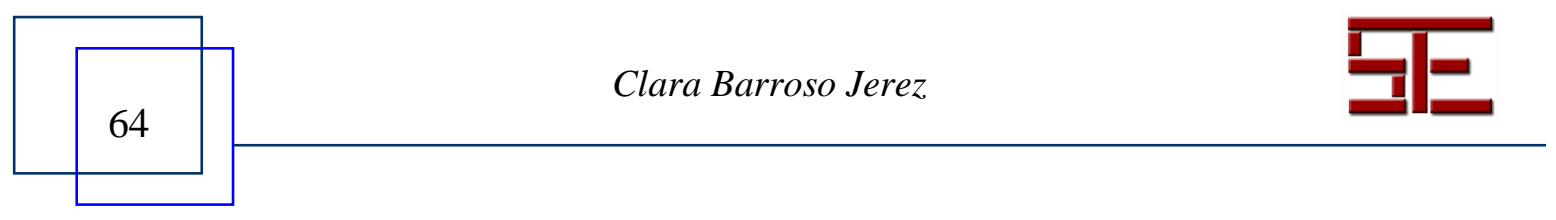




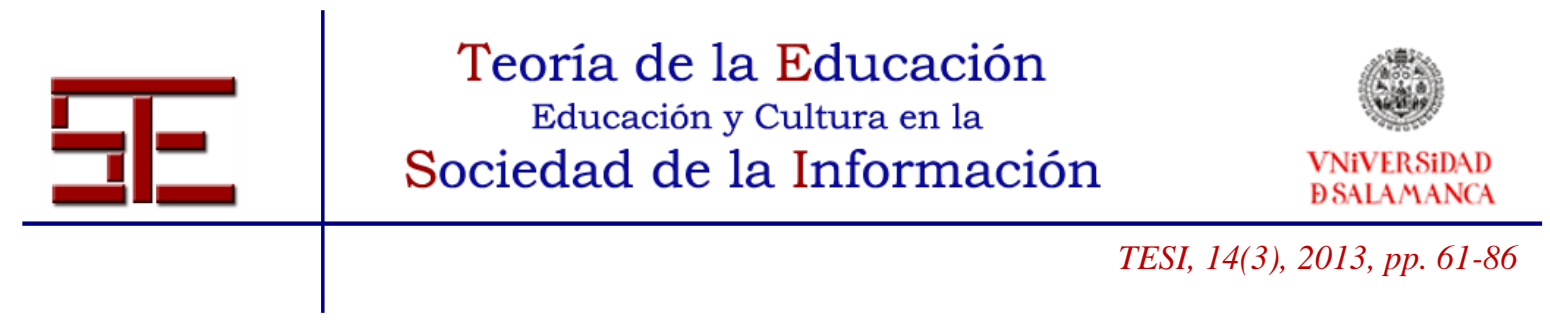

cias, que han de desarrollar los individuos en los ámbitos de dominio de conocimientos previos y de capacidades cognitivas e intelectuales. Estas competencias les deben facultar para llevar a cabo la construcción y asignación de significado a las informaciones a que puedan acceder utilizando el entorno digital, haciendo posible que el acceso a la información sea un medio para llevar a cabo su aportación a la sociedad del conocimiento.

\section{CONSTRUCCIÓN DE SIGNIFICADOS: PERCEPCIÓN, CONOCIMIENTO Y CONTEXTO}

La construcción de la percepción no es exclusivamente un proceso orgánico individual, en ella están involucrados los significados asociados a los estímulos orgánicos que son compartidos por la sociedad a que pertenece el individuo. En otras palabras, el significado se atribuye mediante las experiencias compartidas en el mundo real. Por ejemplo, para los Inuit 'nieve' carece de significado. En el lenguaje Inuit lo significativo es referirse a 'nieve en el suelo', 'nieve derretida', 'nieve en el aire', etc. Estos conceptos, y la percepción de estos hechos, son significativos en su entorno porque de ello depende su supervivencia en el Ártico. Asignar estos diferentes significados, aprender a construir la percepción que les permite la distinción de los estímulos orgánicos, les permite evaluar acciones posibles en cada caso y compartir estos significados les permite la interacción social y la toma de decisiones sobre acciones posibles y acciones adecuadas. En consecuencia, el primer nivel de construcción de significados está vinculado al desarrollo de la percepción de los objetos y eventos del entorno con que nos relacionamos directamente.

\subsection{El conocimiento individual: experiencia en el contexto}

Inicialmente el mundo cognitivo de un individuo tiene pocas categorías y muy difusas, es la información que acumula mediante la experiencia con los objetos y eventos (físicos y sociales) que le rodean, lo que hace que esas categorías iniciales se refinen en otras más precisas asignando significados concretos. Para llevar a cabo el refinamiento de las categorías iniciales, los humanos utilizan el conocimiento del contexto en que pueden ser utilizadas dichas categorías, estableciendo los grados de validez en la asignación de significados posibles, significados probables o significados seguros.

Desde la óptica individual, a medida que vamos teniendo experiencia del mundo, ampliando el acervo de entornos y objetos que podemos percibir, nuestro cerebro amplía la capacidad para establecer nuevas categorías mediante nuevas etiquetas lingüísticas a las que se asocian significados compartidos por el resto de los humanos con que nos relacionamos. De este modo podemos designar objetos y eventos percibidos orgánicamente, y podemos representar intelectualmente objetos o acontecimientos no directamente per-

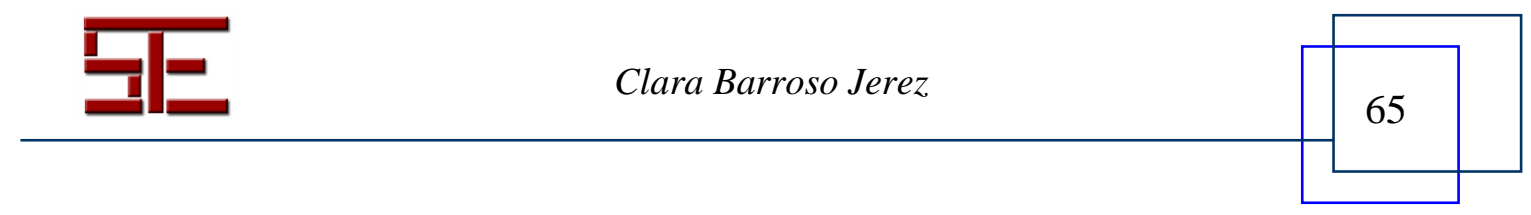




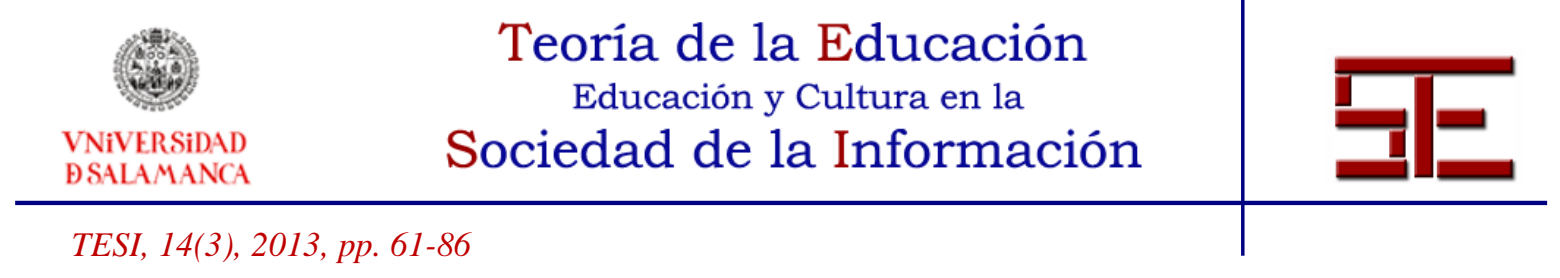

cibidos por los órganos sensoriales. Esta construcción de significados será fundamental en el desarrollo del conocimiento. ${ }^{1}$

Ahora bien, la asignación de significado no se produce fuera del contexto de interpretación. Así, para la mayoría de los individuos no especialistas, la etiqueta 'martillo' posee un significado bastante preciso y podemos pedir que nos den un martillo esperando que nos den lo solicitado; sin embargo un especialista requerirá más información para saber si nos referimos a un martillo para carpintería, un martillo para espeleología, un martillo para geología, un mazo, etc. (olvidemos que los pianos también poseen macillos, también denominados martillos). En este caso, el especialista puede aceptar que el significado seguro de nuestra solicitud se corresponde con un objeto de cierta dureza, con mango (más o menos) ergonómico cuya funcionalidad es percutir. Pero bajo esta definición caben muchas posibilidades. Para un especialista es posible que estemos solicitando un martillo de carpintero con una cabeza de alta dureza, pero también es posible que pidamos una herramienta con cabeza de menor dureza con que poder testar los reflejos de un paciente; es posible que nos refiramos a un martillo hidráulico, etc. lo único seguro es que se trata de un objeto para golpear.

El contexto en que se produce una información va a ser un elemento fundamental para establecer la validez de la asignación de significado de la misma. En el ejemplo anterior, se puede utilizar información adicional (nada pertinente, inicialmente, con la herramienta martillo) para establecer con cierto grado de certeza a qué tipo de objeto nos estamos refiriendo. Así si el especialista percibe el atuendo del solicitante, puede reducir el universo de los significados posibles al universo, más restringido, de los significados probables. Del mismo modo, ingresando informaciones sobre el tipo de uso que se quiere dar al 'martillo' puede incrementar el grado de certeza sobre qué es lo que solicitamos; así la funcionalidad y/o el atributo del objeto permitirá asignar, con mayor precisión, el significado de la etiqueta 'martillo' que estamos utilizando. Este ejemplo muestra que la capacidad para asignar significados precisos a las etiquetas linguiísticas está vinculada a la capacidad de utilización de la información que provee el contexto para establecer el grado de validez asignado al significado atribuido.

El uso de la información que proviene del contexto percibido, permite que podamos emplear una misma etiqueta lingüística con significados diferentes dependiendo del contexto en que nos situemos. Así 'maceta' es un gran martillo si el contexto en que nos situamos es el contexto de la construcción; mientras que si nos situamos en un contexto de jardinería estaremos designando un recipiente para plantar vegetales.

Lo afirmado sobre la atribución de significado de una etiqueta lingüística, asociada a un objeto, puede generalizarse al significado atribuido a un acontecimiento. El contexto en que se producen un acontecimiento permite designar un evento utilizando la informa-

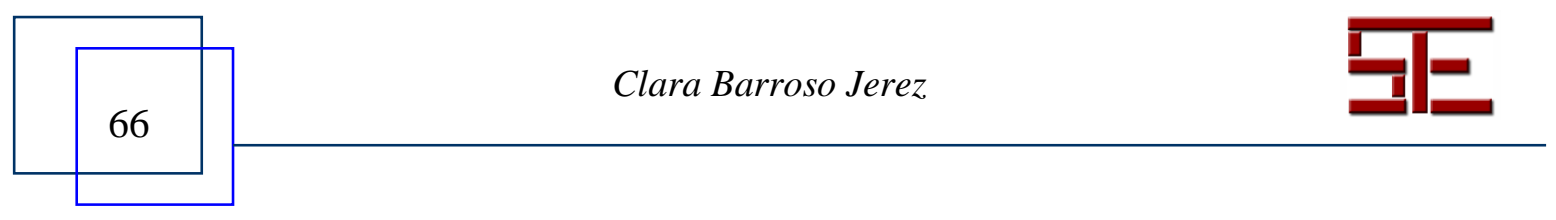




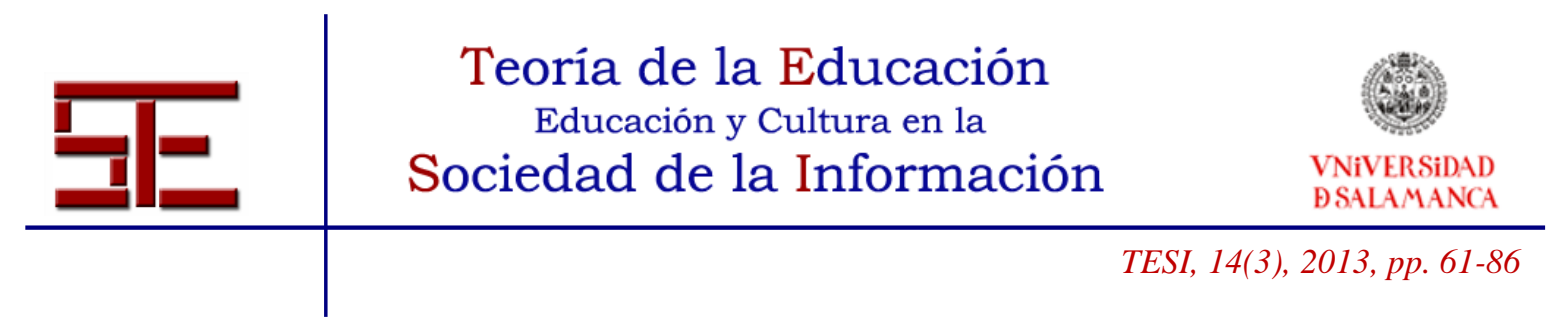

ción sobre el contexto percibido. De este modo construimos el significado de cada evento asignando un significado preciso del acontecimiento que resulte coherente con dicho contexto. Así el acontecimiento 'combate cuerpo a cuerpo' puede ser interpretado como un evento deportivo o como un evento de agresión, dependiendo del contexto percibido en que sucede dicho acontecimiento.

Por tanto la asignación de significados precisos está necesariamente vinculada a la capacidad para percibir o conocer los contextos en que se construyen y utilizan socialmente los significados. La experiencia de cada sujeto incrementa sus facultades para ampliar y diversificar el acervo de significados que es capaz de utilizar, al diversificar y ampliar la cantidad de contextos en que construye y comparte socialmente significados.

\subsection{La experiencia y la construcción de significados}

Como se ha indicado, en la construcción de significados, la experiencia supone no sólo la capacidad de observar el mundo y acumular informaciones. La construcción de significados pertinentes (posibles, probables o seguros) exige que el individuo posea las competencias y posibilidades reales de ser intelectualmente activo para reconocer el contexto en el que contrastar la validez de la asignación de significado de entre los significados potencialmente atribuibles. ${ }^{2}$

El recurso que los humanos utilizan para construir sus acervos de significados descansa en la capacidad para asignar etiquetas simbólicas consistentes con experiencias previas, incorporando estas experiencias a los procesos de análisis del contexto en que se producen. Así, podemos interpretar un acontecimiento de lucha como un evento deportivo, a pesar de no conocer dicho tipo de lucha, por la relación heurística que nos permite hacer corresponder el contexto (instalación deportiva, acontecimiento festivo, etc.) en que se produce ese evento, con otros previamente conocidos.

\footnotetext{
Los símbolos son existencias físicas y sensoriales particulares, lo mismo que cualquier otra cosa. Sólo son símbolos en virtud de lo que sugieren y representan, es decir, de los significados [...] Representan estos significados para cualquier individuo siempre que haya tenido 'experiencia' de alguna situación en la cual estos significados fueran realmente pertinentes. Las palabras pueden destacar y preservar un significado tan sólo cuando el significado se ha visto previamente implicado en nuestro trato directo con las cosas (Dewey, 2007, 237). ${ }^{3}$
}

Así mismo, los humanos utilizan las experiencias previas en otro proceso especialmente valioso: el que se vincula a situaciones en que la información explícita sobre un evento percibido es incompleta o imprecisa. Conociendo las características del contexto en que se producen los eventos, la experiencia nos permite construir significados. Mediante la contextualización de la información somos capaces de indicar qué tipo de información utilizamos (sensorial, cognitiva, intelectual, etc.). Más aún, el contexto también permite

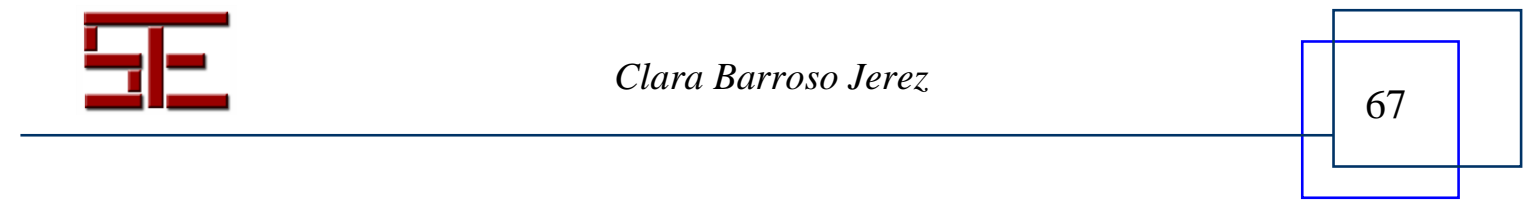




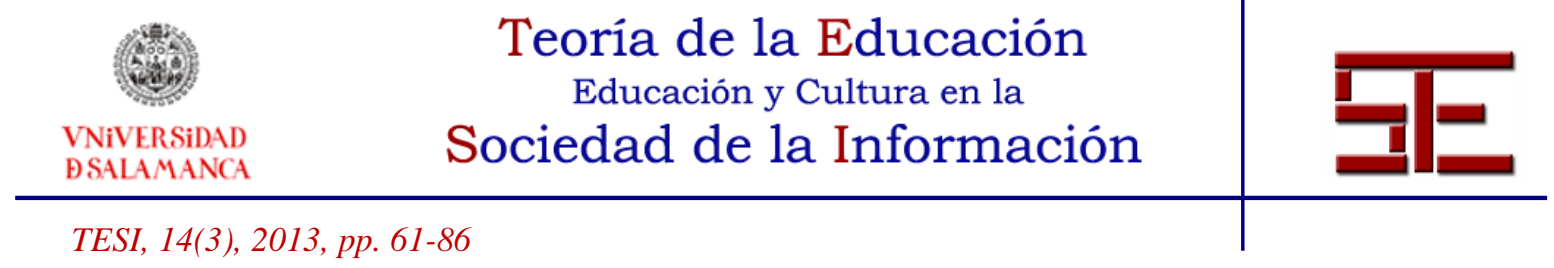

que interpretemos si esa información puede ser incierta, imprecisa, incompleta, vaga, inexistente, etc. y este valor estimado hace posible el que se atribuya validez a un significado. Finalmente el contexto nos permite asignar grados de validez a los significados que podamos estar considerando y decidir si cada significado pertinente es posible, probable o seguro.

\title{
3. CONTEXTO Y PERCEPCIÓN: CONSTRUCCIÓN DE SIGNIFICADOS EN LA EXPERIENCIA
}

Tal como se ha apuntado, la percepción está vinculada a la construcción de significados. En cada idioma cada palabra, cada etiqueta simbólica, tiene asociados significados establecidos; en ocasiones estas etiquetas son polisémicas y van a ser las sentencias lingüísticas en que se usan las que determinarán cuál de los significados posibles corresponde con el contexto de información suministrado por la sentencia lingüística. A su vez, la percepción de la realidad determina lo que, en cada momento, o en cada contexto, adquiere un determinado significado. J. Dewey muestra esta asignación de significado mediante el siguiente ejemplo:

\begin{abstract}
Si una persona entra repentinamente en nuestra habitación y exclama "Paper!", hay varias alternativas posibles. Si no entendemos la lengua, sólo representa para nosotros un ruido que puede actuar como estímulo o irritación física. Pero el ruido no es un objeto intelectual, carece de valor intelectual. [...] Si, en primer lugar, el grito es el acompañamiento usual de la entrega del periódico de la mañana, el sonido tundra significado, contenido intelectual, lo comprenderemos. O si, en Segundo lugar, estamos ansiosos esperando recibir algún documento importante, podemos suponer que la exclamación significa un anuncio de su llegada. Si, en tercer lugar, entendemos la lengua, pero nuestros hábitos y expectativas no sugieren ningún contexto, la palabra tiene significado pero no el acontecimiento (Dewey, 2007,145-146). ${ }^{4}$
\end{abstract}

En la construcción de la percepción del mundo, el contexto en que se producen los hechos o acontecimientos opera como referente que delimita los universos de discurso y de realidad, en la asignación de significado a las informaciones (sentencias lingüísticas) que utilizan los humanos. Así, el contexto opera estableciendo límites a los significados asociados a una palabra o una declaración lingüística que, de otro modo, podrían ser interpretadas de muy diversas formas.

El fundamento de la asignación de significado (contexto) puede ser una disciplina intelectual, una realidad social o una realidad física.

En el caso del contexto de interpretación vinculado a una disciplina, viene determinado por el dominio de conocimientos de la misma. Por ejemplo, la etiqueta lingüística 'revolución' puede estar vinculada a la Historia, la Sociología, la Geometría o la Física. Sin

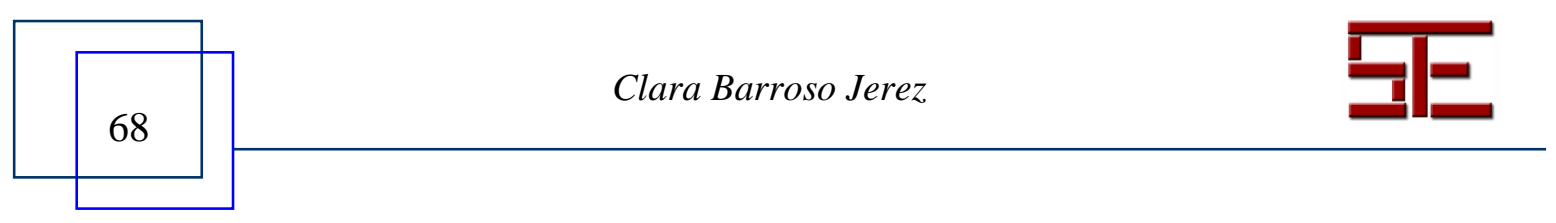




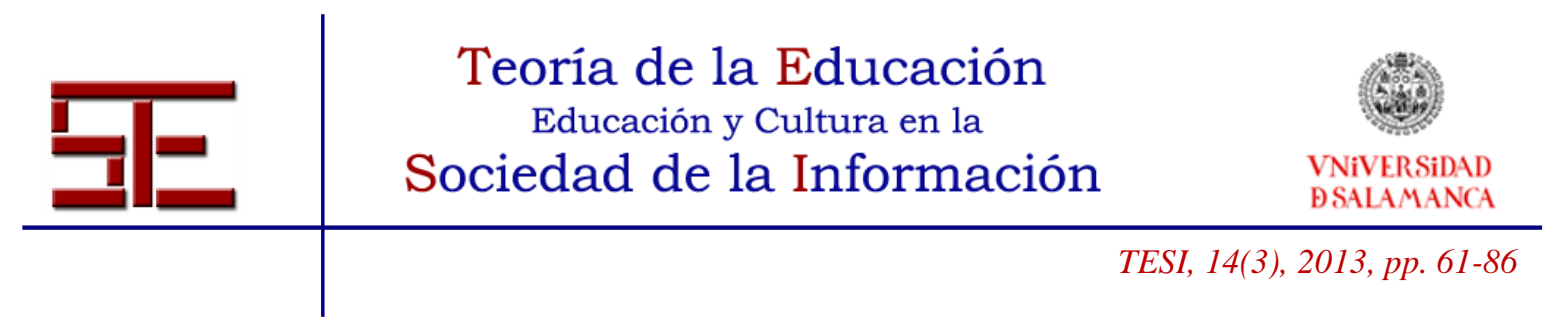

considerar el contexto disciplinar en el que nos situamos, la afirmación "esto es una revolución" carecerá de significado.

En el caso habitual de una realidad social, el contexto puede ser representado por el universo de discurso que comparten los individuos de una comunidad. Así en el contexto social de los profesores, las referencias a las TIC se asocia a unos determinados procedimientos y herramientas de apoyo a la enseñanza. Cuando un profesor afirma "yo utilizo las TIC", interpretamos que se refiere al tipo de actuaciones que lleva a cabo en el aula (física o virtual) en su desempeño docente. En un contexto más amplio cuando un individuo afirma "yo utilizo las TIC", interpretamos que puede referirse a que utiliza la banca o el comercio electrónico, que usa habitualmente el correo electrónico, que usa su tiempo libre en juegos on-line, etc. Dependiendo del contexto social en que nos situemos el significado de usar TIC se refiere a un conjunto de prácticas bien diferentes.

En el caso de una realidad física, el contexto de interpretación puede estar asociado a la funcionalidad de un objeto. Un martillo puede usarse en pruebas diagnósticas para conocer el estado de reflejos de un paciente. También nos referimos a un martillo en determinadas pruebas físicas. Así el contexto de interpretación de la etiqueta lingüística 'martillo' depende de si el uso que se quiere dar a ese objeto pertenece al conjunto de instrumentos utilizados por un atleta o por un médico. El 'martillo' utilizado por un médico en sus exploraciones diagnósticas no se corresponde con el objeto 'martillo' utilizado por el atleta. La funcionalidad de ambos difiere significativamente y nadie suministrará al atleta el martillo de un médico para llevar a cabo sus pruebas físicas. ${ }^{5}$ Es más, si podemos percibir la indumentaria del usuario potencial del martillo, estamos en condiciones de suministrarle, con mayor exactitud, el objeto que nos solicita.

Dicho en otras palabras, los humanos utilizan informaciones (en ocasiones accidentales) que provienen del contexto para reducir el nivel de incertidumbre en la asignación de significado a etiquetas y proposiciones linguiísticas. En palabras de Dewey: "Puesto que todo saber, incluso toda investigación científica, intenta revestir de significado a todas las cosas y todos los acontecimientos, esto es, a comprenderlos, siempre procede a sacar de su aislamiento a la cosa que hay que investigar" (Dewey, 2007, 146). ${ }^{6}$

El dominio de capacidades heurísticas permite desarrollar procesos por los que, en ausencia de información exhaustiva sobre el evento percibido, podemos 'completar' la información a que accedemos permitiendo la asignación de significados, con cierto grado de certidumbre, incorporando información sobre el contexto en que se produce dicho evento. Mediante la contrastación del valor social del significado atribuido, se puede asociar un grado de certeza a dicho significado; dicha contrastación intersubjetiva permite establecer si el significado atribuido corresponde a lo imposible, lo posible, lo probable o lo seguro en el contexto del conocimiento socialmente compartido. ${ }^{7}$

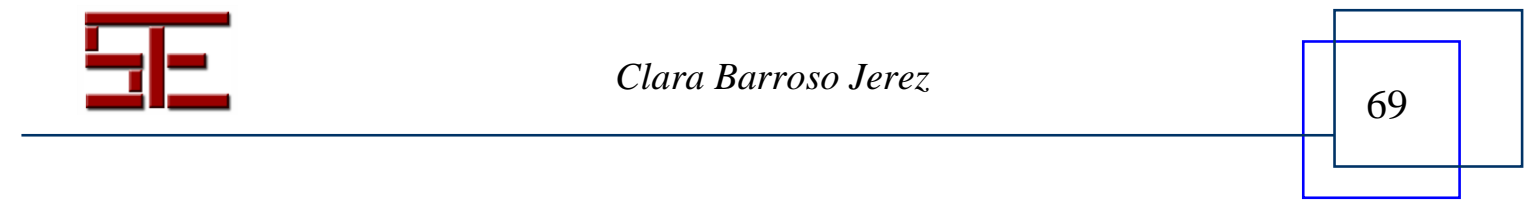




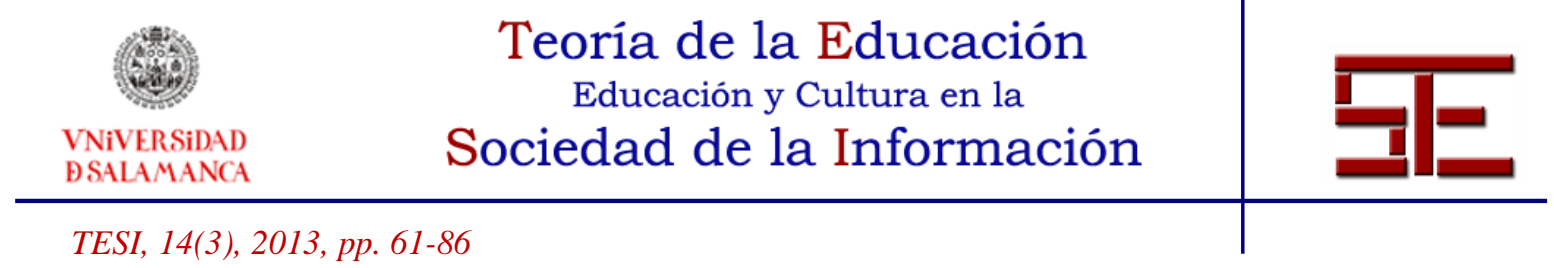

\subsection{El conocimiento especializado y los contextos de significado}

Por último, el dominio de etiquetas lingüísticas compartidas por comunidades especializadas (entornos científicos) permite desarrollar representaciones significativas de realidades no inmediatas, realidades que pertenecen a contextos remotos o contextos abstractos. La mayor parte de las realidades sobre las que reflexiona la ciencia son realidades que superan las experiencias que, como organismos, podemos percibir. Las formas de vida microscópicas o no vinculadas a nuestro entorno vital (bacterias, formas de vida de los fondos abisales o en cráteres); entidades abstractas como teoremas o reglas lingüísticas; realidades macroscópicas, como las galaxias o ínfimas como los elementos atómicos; procesos que se producen en escalas temporales que superan la experiencia directa por producirse en escalas temporales lentas (crecimiento, evolución de especies) o rápidas (reacciones químicas); propiedades que se pueden medir indirectamente pero que no son sensorialmente perceptibles (conductividad de la electricidad o del calor) son ejemplos de estas realidades no directamente perceptibles.

El dominio del contexto científico de referencia, determina el universo de significados pertinentes. Así, la etiqueta linguística 'plasma' posee significados diferentes dependiendo de si nos situamos en el contex to de la Biología o la Medicina o si se utiliza en el contexto de la Física, la Astrofísica o la Ingeniería Electrónica. Nuevamente va a ser el dominio del contexto de conocimiento en que se utilice la etiqueta lingüística, en el que permita dotar de significado válido a la misma, y establecer una asignación precisa de su significado. En el ámbito científico, el acceso y utilización de información significativa está fuertemente vinculada al contexto de interpretación disciplinar, la experiencia acumulada en dicho contexto (dominio de la disciplina, dominio del universo de discurso especializado, capacidad para diferenciar lo que pertenece a dicho discurso y lo que pertenece a otro universo de discurso, etc.) es lo que permite la construcción de significados precisos, y el acceso y desarrollo del dominio de conocimientos científicos.

Más aún, la validez del significado de conceptos como 'inteligencia humana' puede depender de un contexto más restringido: el contexto teórico en que nos situemos. Así la afirmación Roberto es muy inteligente carece de contenido semántico a menos que compartamos un significado de inteligencia. En el contexto de la Psicología Positivista podremos interpretar que Robert posee un CI superior a 145; en el contexto de la Psicología Cognitiva podremos interpretar que Roberto domina competencias y habilidades que le permiten afrontar con éxito la solución de muchos y diferentes tipos de problemas. Ambos significados atribuidos serán válidos en diferentes contextos teóricos.

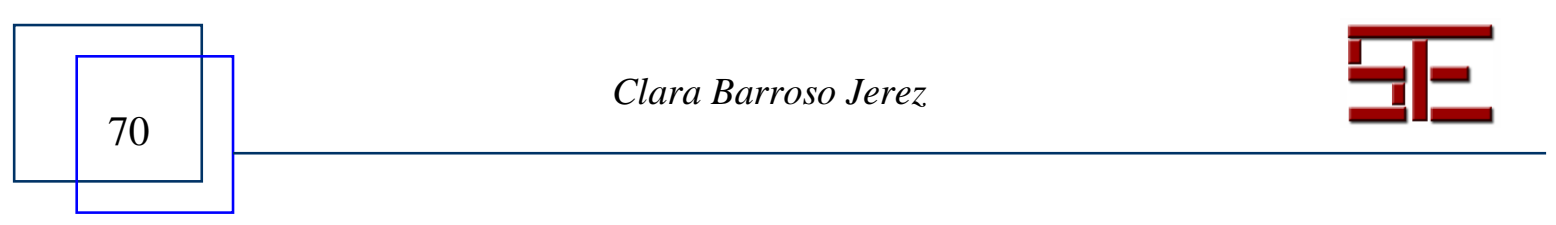




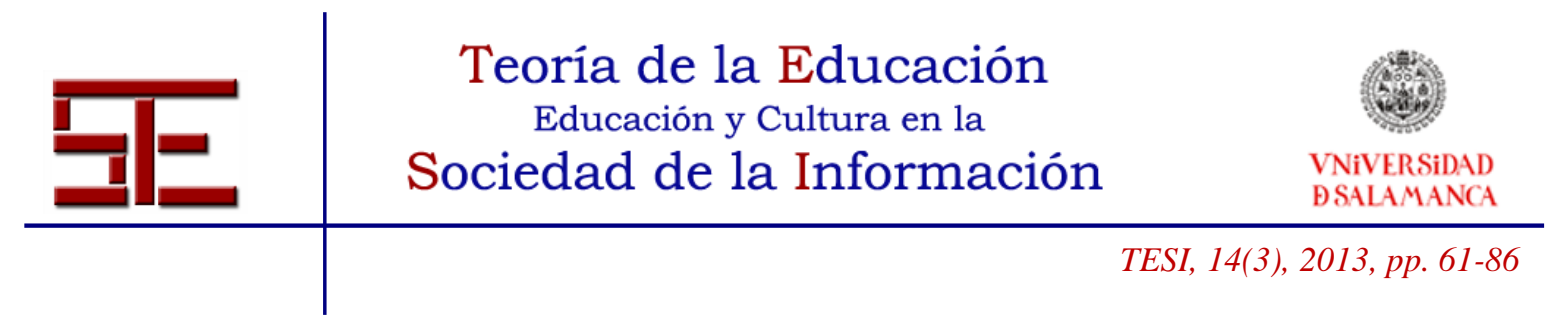

\subsection{El dominio de conocimiento}

Como consecuencia de lo expuesto, se puede afirmar que los humanos utilizan información sobre el contexto para desarrollar procesos de asignación de significado y para atribuir grados de certeza a los significados posibles. En esta atribución, las experiencias previas, las capacidades desarrolladas para dominar procesos de análisis y evaluación de los contextos en que suceden dichas experiencias, y el dominio de contextos disciplinares constituyen el fundamento de la construcción de lo que designamos como 'dominio de conocimiento'. En consecuencia, nuestro dominio de conocimientos contribuye a atribuir a los objetos o eventos (físicos o intelectuales) ante los que nos situamos significados válidos, utilizando para ello las experiencias previas con los mismos u otros que heurísticamente percibamos como similares. Tal como afirma J. Dewey: "Todo juicio, toda deducción reflexiva, presupone alguna carencia de comprensión, una ausencia parcial de significado. Reflexionamos con el fin de apoderarnos del significado total y adecuado de lo que sucede. Si embargo, algo ha de haberse comprendido ya, la mente debe estar en posesión de algún significado que haya dominado, o el pensamiento sería imposible" (Dewey, 2007, 147). ${ }^{8}$

Ahora bien, cómo los humanos utilizan esa experiencia previa no está definido a priori. No hay reglas establecidas que determinen la utilización del acervo de experiencias acumuladas en términos de asociación estímulo-significado mediante inferencias lógicas. Para llevar a cabo la asignación de significados es necesario situar cada estímulo (o información) en el contexto percibido. Más aún, la capacidad de percibir el contexto en que nos situamos, permite la asignación de significados a las informaciones (aun en ausencia de información precisa o completa sobre un objeto o evento) y permite otorgar un grado de certeza a dicho significado. Dependiendo de nuestra capacidad para conocer contextos posibles, en que dicha información puede adquirir significados precisos, podemos determinar el grado de validez que posee la asignación de significado que realicemos. Finalmente, el uso de dicho significado en el contexto social mostrará lo adecuado o inadecuado de nuestra opción; en otras palabras, contrastamos la asignación de significado que hemos llevado a cabo.

\section{INFORMACIÓN，CONSTRUCCIÓN DE CONOCIMIENTO Y ENTORNO DIGITAL}

Los primeros estudios sobre la sociedad de la información hicieron hincapié en el peligro de aparición de lo que se ha denominado la 'brecha digital'. Inicialmente se señalaron los riesgos de una fractura social, originada por factores de tipo socioeconómico, que dividirían a la sociedad de la información entre los individuos con capacidad para acceder a las herramientas y soportes informáticos y los que se verían excluidos. Si bien es cierto que las variables de tipo socioeconómico siguen teniendo un peso específico al

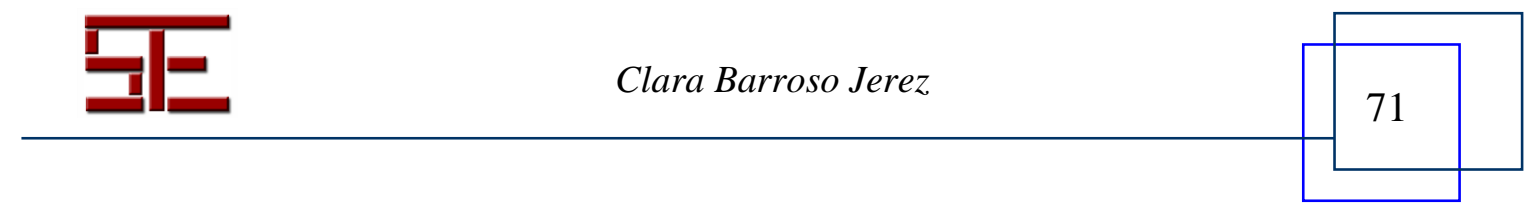




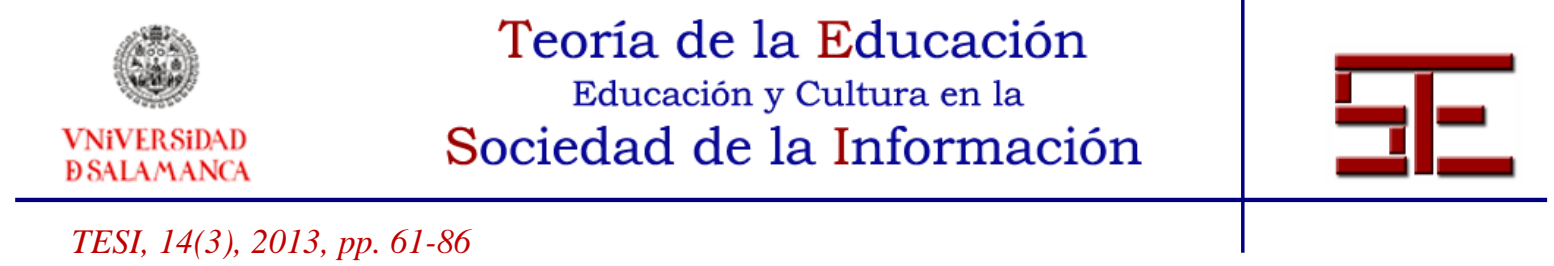

considerar las diferencias de oportunidades en el acceso a la sociedad de la información, el abaratamiento de los costes de hardware y software, ha permitido que, en muchas sociedades, se haya impulsado un consumo generalizado de las herramientas vinculadas al entorno digital. ${ }^{9}$

Las tesis sobre la brecha digital se amplía más adelante para señalar una nueva 'brecha' (esta vez fundamentada en consideraciones demográficas) entre los individuos que pertenecerían a la sociedad interconectada y los que accederían a ella, denominando 'nativos digitales' a aquellos individuos que habrían nacido en la sociedad en la que ya se ha generalizado el acceso al entorno digital, mientras que los individuos nacidos con anterioridad se definirían como 'inmigrantes digitales'. Para los defensores de esta categorización esta cuestión demográfica daría lugar a una división de lo que denominan la sociedad global.

A pesar de estas limitaciones, durante los últimos años del siglo pasado las prospectivas sobre la generalización del uso de las TIC preveían que estos nuevos canales de información impulsarían el desarrollo de más y mejores oportunidades de desarrollo social y personal, asumiendo que la sociedad de la información necesariamente daría lugar a una sociedad informada, una sociedad en la que cada individuo poseería y desarrollaría las capacidades necesarias para ser un miembro activo en la sociedad del conocimiento (Gates, Myhrvold y Rinearson, 1996).

En la actualidad se aborda el análisis de problemas más profundamente vinculados a otro tipo de brecha: los problemas relacionados con el acceso masivo a las fuentes de información y sus efectos en el desarrollo de la sociedad del conocimiento. Desde nuestra perspectiva, si bien se ha generalizado el uso de los recursos informáticos, queda por justificar que la sociedad de la información haya dado lugar a oportunidades reales de desarrollo social e individual; de desarrollo de una sociedad en la que el conocimiento compartido, mediante el uso del entorno digital, haya incrementado las posibilidades y oportunidades de construcción de conocimiento válido y de desarrollo individual y social. Dicho de otro modo, la existencia de la sociedad de la información, no justifica ni garantiza la existencia de la sociedad del conocimiento.

Para abordar la reflexión de lo que supone el entorno digital y su vínculo con la construcción de conocimientos, vamos a explorar algunos aspectos que nos resultan significativos.

Tal como hemos expuesto, la construcción del conocimiento no es sólo deudora de la capacidad para acceder a la información, por encima de ello es la capacidad para atribuir significado válido a dicha información, lo que puede permitir (o impedir) conocer. En segundo lugar, la sociedad de la información se ha definido como aquella que hace

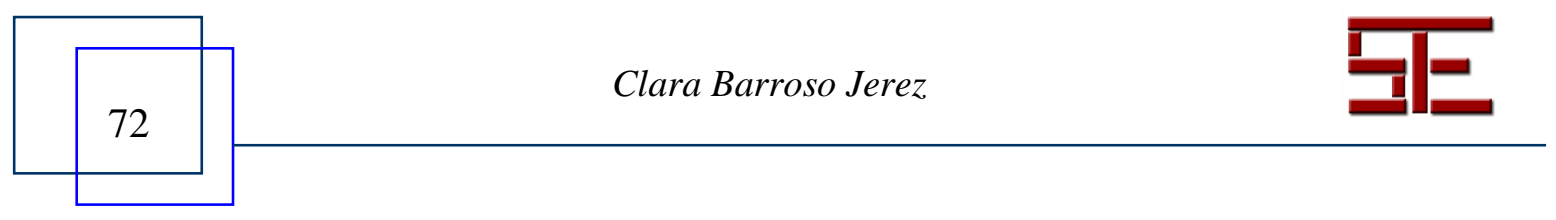




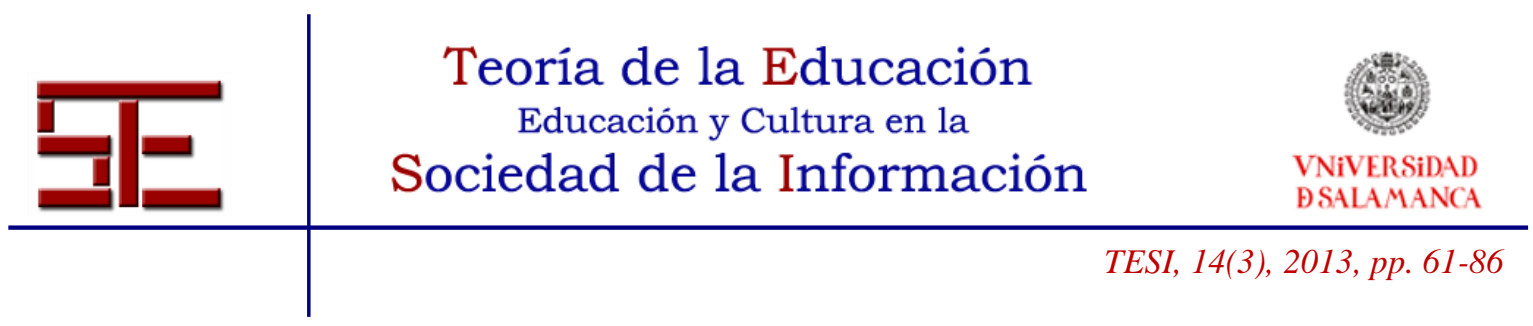

del acceso a la información el instrumento que ha de potenciar la construcción y desarrollo de conocimientos. En tercer lugar, la sociedad de la información, vinculada a las TIC, ha dado lugar a un nuevo 'entorno' -el entorno digital- en que los individuos adquieren y desarrollan nuevos significados. Abordaremos ahora el análisis de esta afirmaciones.

\subsection{El entorno digital y la construcción de significado}

En primer lugar hemos de establecer cuál es el contexto disciplinar en que aparece el 'entorno digital' ya que (tal como se ha indicado) el contexto disciplinar es un referente para la construcción de significado. El contexto disciplinar en que aparece y se desarrolla el entorno digital es el contexto informático. En el mismo son las inferencias lógicas las que determinan, sin incertidumbre posible, la asociación de significados a etiquetas lingüísticas. En el mejor de los casos, (cuando el motor de inferencias utiliza lógica fuzzy) se puede asociar un grado de certeza a la asignación de los significados posibles de cada etiqueta lingüística. Así la asignación de significados se realiza en un mundo cerrado en el que el contexto está previamente establecido o, como señala E. Trillas, es local.

La lógica fuzzy es esencialmente local, dependiente del contexto y viene a ser una representación semántica que violente lo menos posible las estructuras con sintaxis forzada; es flexible, de carácter más bien analógico que digital. Quién sabe si, en el futuro y con su misma evolución hacia el Soft-Computing, la lógica fuzzy no podrá ser una ayuda para comprender y "normalizar" los patrones del hipotético lenguaje del pensamiento que, frente a la sintaxis de los lenguajes ordinarios, parece ser inicialmente semántico (Trillas, 1998, 185).

Como consecuencia, los procesos de asignación de significados en este entorno distan mucho de los procesos típicamente humanos. A diferencia de los procesos humanos, los procesos informáticos están previamente 'programados'. ${ }^{10}$ Operan con una ontología lógica que determinará cuáles son los significados posibles que en ellos se implementan. Por ello, el tratamiento de la información en el entorno digital no se corresponde con el uso que realizan los humanos de la información en el proceso de asignación de significados y construcción de conocimiento.

En el caso de los humanos la experiencia perceptual del contexto va actualizando tanto las informaciones de que se dispone, como las posibilidades de utilización de las mismas y las posibilidades de asignación de significados; en el caso de los sistemas informáticos existe un modelo previamente programado que determina el tratamiento de la información y la asignación de significados.

Distinguir el proceso lógico de tratamiento de la información en el contexto digital y reconocer las diferencias con los procesos humanos (en ocasiones muy ilógicos), permi-

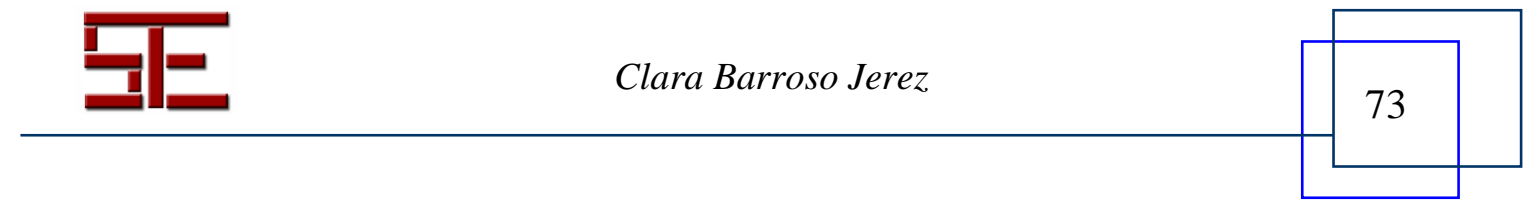




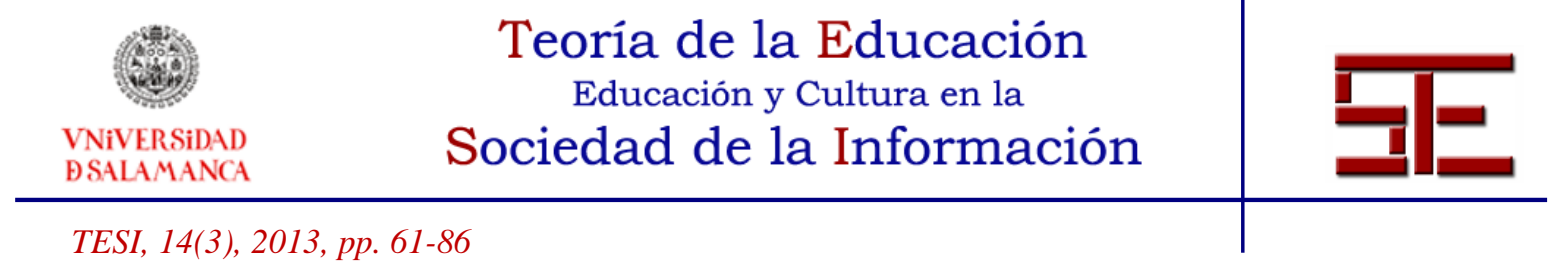

tirá comprender el valor relativo de las informaciones a que se accede en el entorno digital. Este hecho es relevante si se desea que los usuarios del entorno digital comprendan el valor de los procesos mediante los que acceden a la información y el significado atribuido a la misma. En este cometido, parece que la brecha entre los inmigrantes digitales y los nativos digitales es inexistente tal como muestran las investigaciones de van Dijk y van Deursen (van Dijk y van Deursen, 2010)

\subsection{Contexto de información y entorno digital: indagar y buscar, dos estrategias para usar la información y construir conocimiento}

Para establecer el fundamento de nuestro análisis vamos a distinguir entre indagar y buscar información. Cuando indagamos estamos en situación de buscar información significativa, es una búsqueda orientada por objetivos de conocimiento que responden a la necesidad de resolver una duda. "La exigencia de solución de un estado de perplejidad es el factor orientador y estabilizador de todo el proceso de reflexión. Allí donde no se trata de resolver un problema o superar una dificultad, las sugerencias se suceden al azar" (Dewey, 2007, 29). ${ }^{11}$ En este sentido afirmamos que constituye una actividad de pensamiento racional.

El proceso de indagación de los humanos trasciende al mero tratamiento de la información concreta y puntual. En la indagación se desarrolla la búsqueda de información, se atribuye un valor respecto del problema que ha provocado la búsqueda y se juzga su adecuación para solucionar el problema. Al incorporar el contexto como fuente de significado, la experiencia es parte sustancial de la búsqueda de información y de utilización de la misma en la construcción de conocimiento y solución de problemas. El significado atribuido a la información, el juicio de lo relevante o irrelevante que es (en el contexto del problema que sugiere la indagación) es lo que le lleva a utilizar dicha información, en la mayoría de los casos, eficientemente.

En el entorno digital (si bien se van desarrollando e implementado estrategias que persiguen acercar las ontologías de los motores de búsqueda de información a los requerimientos de los usuarios humanos) los procedimientos automáticos de búsqueda de información se fundamentan en estimaciones estadísticas sobre etiquetas lingüísticas previamente utilizadas por los usuarios en la búsqueda de información. Así los procedimientos automáticos de búsqueda de información adopta estrategias de captura de información, sobre procedimientos previos de un usuario, como asunciones previas para optimizar ulteriores búsquedas. Dicho en otras palabras, se modeliza al usuario de acuerdo con el ingreso, en el motor de búsqueda, de etiquetas que dicho usuario previamente ha utilizado. "Estos sistemas normalmente adoptan asunciones sobre el contexto de búsqueda en los que operan y los usuarios que los usan" (Marchionini y White, 2010, $227)^{12}$

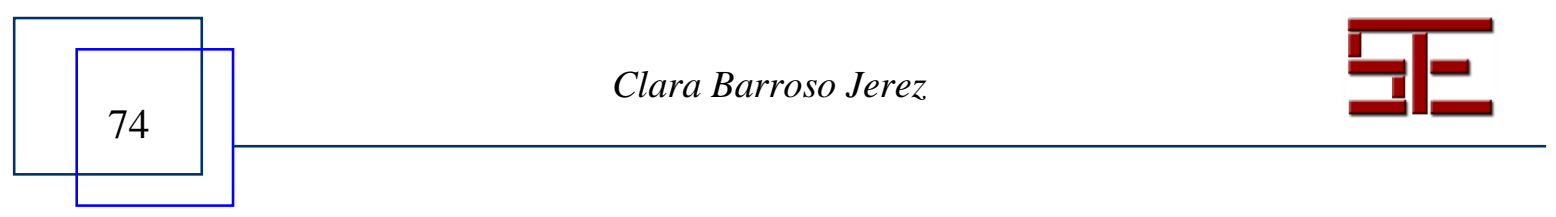




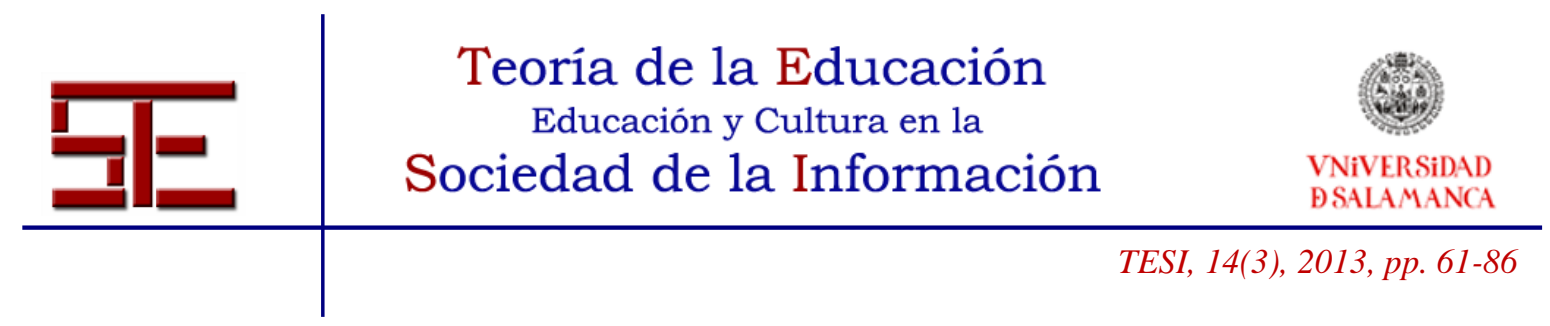

Buscar información, en el entorno digital, no necesariamente está orientado por un proceso racional de indagación. En numerosas ocasiones responde a una captura aislada (sin referente a un contexto de interpretación) carente de significado racional. Más aún, lo motores de búsqueda utilizan estrategias no siempre conocidas o comprendidas por los usuarios del entorno digital.

Los sistemas de búsqueda normalmente permiten expresiones booleanas y ofrecen operadores de búsqueda avanzada como las comillas que pueden incrementar la precisión de resultados de búsqueda pero [su uso] debe ser aprendido e incluido en los enunciados de búsqueda. Sin embargo, muchos usuarios ignoran estos operadores, principalmente porque su uso no es público y la interface de generación de consultas que los usa está oculto a un inicial punto de vista. Como resultado, muchos usuarios carecen de las habilidades adicionales requeridas para formular búsquedas bien definidas (Marchionini y White, 2010, 211). ${ }^{13}$

La distinción entre estos dos modos de acceder a la información (búsqueda de información en términos de indagación y búsqueda de información automática) es relevante en la medida en que, sin conocimiento de los límites de lo que son los procesos programados para buscar información, se puede atribuir al proceso automático el valor un proceso humano de indagación.

En el caso de los humanos, la indagación es un proceso en el que los individuos no sólo adquieren información, también van comprobando la validez de las informaciones y los significados que a éstas atribuyen mediante la contrastación de la coherencia de estos significados con el contexto social, físico e intelectual en que se sitúan. Esto facilita la asignación de grados de certeza (validez) al significado atribuido.

En el caso de la búsqueda automática de información, se omite la relevancia del contexto de significado. Dicho de otro modo, el motor de búsqueda automática, se atribuye el valor del contexto de contrastación. Sin ser conscientes de este hecho, los usuarios pueden estar subordinados a los imperativos de la industria de la información y las comunicaciones. La capacidad para establecer lo significativo de una etiqueta lingüística, o de un entorno de búsqueda automática de información, asociada a un problema, afecta a los resultados que los usuarios del contexto digital obtienen y, en consecuencia, a la validez y significado atribuido a las informaciones a que acceden para construir conocimiento.

Debido a que la cualidad de la consulta directamente afecta a la calidad de los resultados de la búsqueda, se debe prestar especial atención a la precisión en la obtención de la descripción del problema del usuario. La formulación de una consulta requiere dos tipos de representación; una representación semántica del vocabulario que el usuario utiliza para expresar la tarea, dentro del vocabulario utilizado por el sistema para lograr la entrada al contenido; y una acción de representación de las estrategias y tácticas que el usuario considera mejores para desarrollar la tarea en las reglas y características en lo que la interface del sistema permite. Los enunciados de búsqueda que surgen de la formulación de la consulta son sólo aproximados o acuerdos sobre la in-

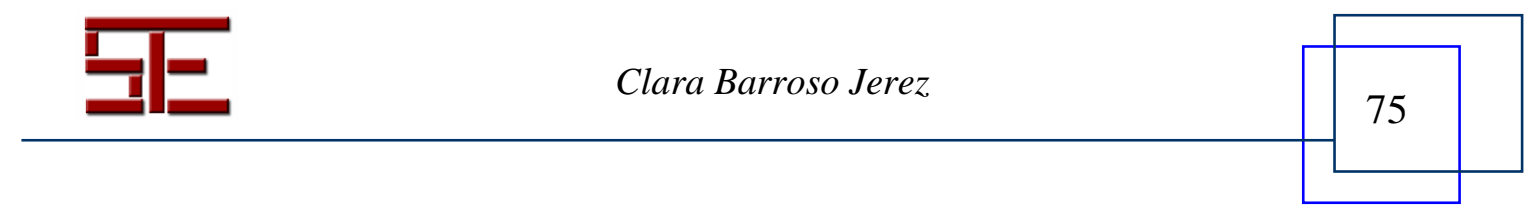




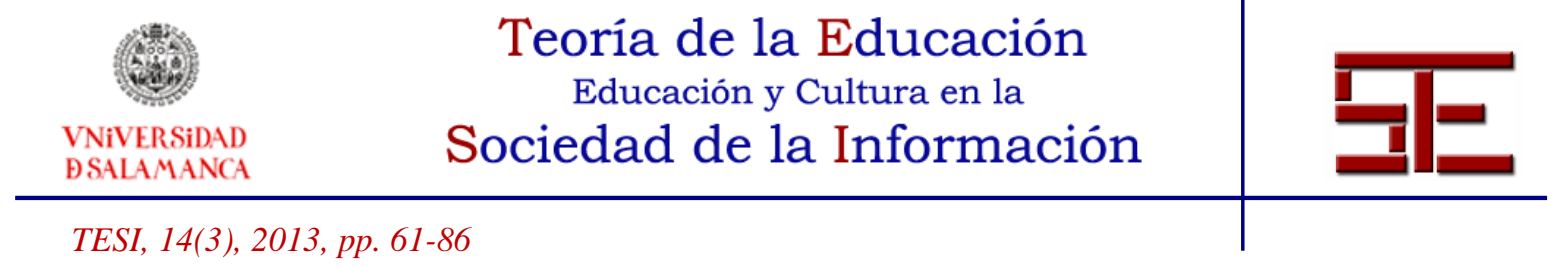

formación necesaria y pueden no llegar a la descripción necesaria para recuperar documentos relevantes simplemente porque el vocabulario del usuario y del sistema difieren grandemente (Marchionini y White, 2010, 210). ${ }^{14}$

En el uso del entorno digital es habitual que, en la búsqueda de información, los usuarios ingresen etiquetas de acuerdo con sus expectativas cognitivas, de acuerdo con lo que, en su dominio de conocimientos, sea significativo; no obstante, el motor de búsqueda automática puede contener una ontología no coincidente con el dominio del usuario. Un ejemplo clásico son los enlaces de tipo "preguntas frecuentes" o "enlaces de interés"; en numerosos casos este tipo de enlaces carecen de interés para los usuarios cuyas dudas transgreden el valor estadístico de las dudas o requerimientos de la media.

\begin{abstract}
La búsqueda de información considerada como una actividad humana es parte de la actividad general de la vida. Puede tener lugar en pocos segundos o a lo largo de toda la vida, puede ser muy puntual, o puede estar integrada en el ritmo de la vida cotidiana. Coloquialmente, búsqueda de información y buscar información son sinónimos; sin embargo nosotros hacemos la distinción de que la búsqueda de información es una actividad únicamente humana mientras buscar información puede ser realizada por máquinas y humanos Aunque la búsqueda de información es impulsada por las necesidades y los comportamientos humanos, y son por lo tanto muy variables, hay varias subactividades comunes que pueden ser apoyadas por un buen diseño técnico. En última instancia, los sistemas de búsqueda bien diseñados, tienen por objeto apoyar estas subactividades y el proceso global de búsqueda de información. En la actualidad, la mayoría de los sistemas de búsqueda se centran en una o unas pocas de estas subactividades llevadas a cabo por las máquinas y los seres humanos. En la medida en que son compatibles con otros tipos de aplicaciones de procesamiento de información, que apoyan los objetivos más ámplios que motivan la búsqueda, el procesamiento de información es adecuado, con independencia de que buscamos sistemas más integrales en el futuro (Marchionini y White, 2010, 207). ${ }^{15}$
\end{abstract}

En consecuencia, el uso del entorno digital como facilitador del acceso a información significativa debe llevarse a cabo comprendiendo los procedimientos que la búsqueda automática suponen. Comprender el hecho de que la información suministrada por un motor de búsqueda es relativa a la ontología que se representa en el mismo, permitirá valorar la relevancia de las informaciones descubiertas y asignar un significado y un valor a dicho significado. Los usuarios han de ser conscientes de los límites de la búsqueda automática de información y han de ser capaces de evaluar la información a la vez que valoran el procedimiento y el contexto en el que han capturado dicha información. Sólo así estarán en situación de utilizar la información obtenida con criterios racionales que hagan posible la construcción de conocimiento.

\title{
4.3. Habilidades, capacidades y construcción de conocimiento en el entorno digital
}

En la actualidad, numerosos estudios abordan, el discurso sobre la capacidades necesarias para hacer un uso adecuado del entorno de información vinculado a las TIC (EshetAlkali y Amichai-Hamburger, 2004; Marchionini y White, 2010; van Deursen y van

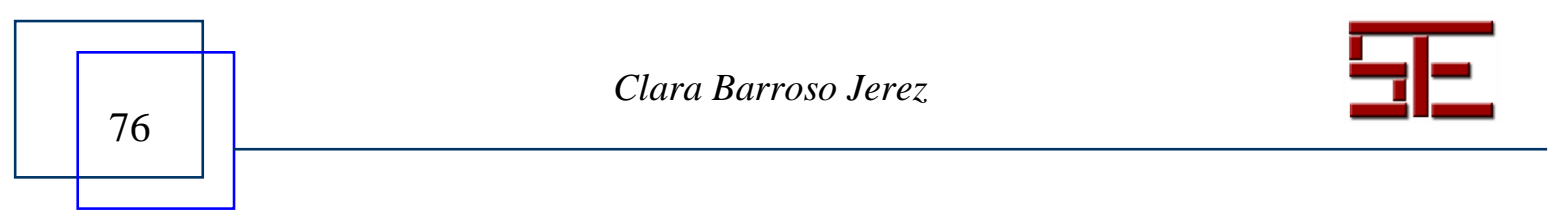




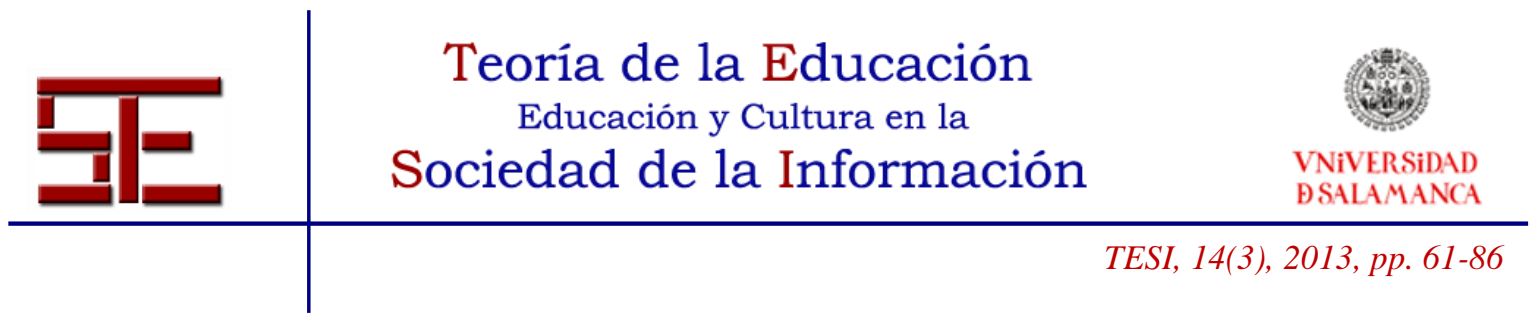

Dijk, 2009; Bonfadelli, 2002). Básicamente, los investigadores actuales orientan sus estudios aceptando que es necesaria una 'alfabetización digital' que permita hacer un uso adecuado del entorno digital. Qué supone dicha 'alfabetización' en, términos de habilidades y competencias es el problema más relevante a la hora de vincular sociedad de la información con sociedad del conocimiento.

La alfabetización digital es el conjunto de perspectivas desde las que nos exponemos a los medios de comunicación e interpretamos el significado de los mensajes que encontramos. Construimos nuestras perspectivas desde estructuras de conocimiento. La estructura de conocimiento forma las plataformas en las que nos situamos para ver el polifacético fenómeno de los medios de comunicación: sus negocios, sus contenidos y sus efectos en los individuos e instituciones [...] Cuantas más personas utilicen esas estructuras de conocimiento en experiencias conscientes, estarán más capacitadas para usar sus experiencias en los medios de comunicación para lograr sus propios objetivos, y estarán más capacitadas para evitar los altos riesgos de efectos negativos (Potter, 2004, 58-59). ${ }^{16}$

La alfabetización digital requiere más que la superación de las brechas digitales por razones socioeconómicas y sociodemográficas. Así las actuales investigaciones se orientan hacia la definición de qué habilidades son necesarias para obtener esa alfabetización digital. Inicialmente se pueden considerar las siguientes habilidades básicas:

-Definición del problema o tarea que determina la búsqueda de información

-Estrategias de búsqueda de información

-Localización y acceso a la información necesaria

-Utilización de la información

- Capacidad de síntesis

- Capacidad de evaluación de la información considerada

En un plano más general de categorización de estas habilidades, van Deursen y van Dijk (van Deursen y van Dijk , 2009) indican:

- Habilidades operativas, que se relacionan con la capacidad realizar actividades básicas (seleccionar un link, guardar un documento PDF, cargar ficheros, añadir una localización web en 'favoritos', etc.)

-Habilidades formales, relacionadas con la capacidad de considerar múltiples links en un menú, sin desorientarse en la tarea de búsqueda, pudiendo utilizar diferentes recursos de navegación y valorando el resultado de la búsqueda

- Habilidades de información, vinculadas a la capacidad de encontrar información en un entorno concreto y realizar tareas en un entorno web.

-Habilidades estratégicas, vinculadas al objetivo de incrementar la capacidad de utilización significativa de la información encontrada

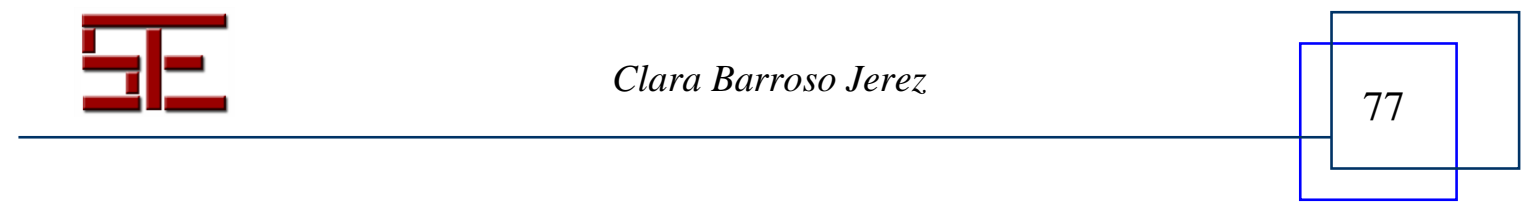




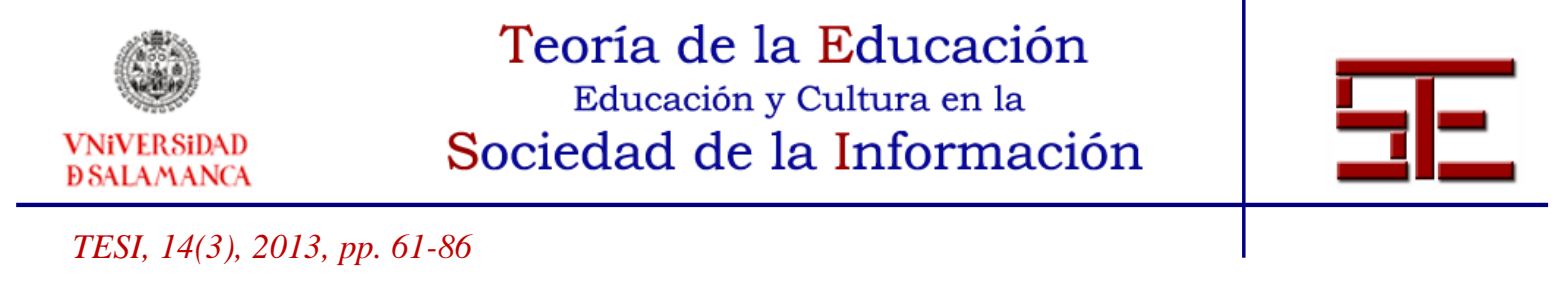

En un plano más teórico, Eshet-Alkalai y Amichai-Hamburger (Eshet-Alkalai y Amichai-Hamburger, 2004) proponen las siguientes competencias:

- Alfabetización visual: capacidad para 'leer' instrucciones en formato gráfico

-Alfabetización de edición: capacidad para generar nuevos materiales significativos utilizando la información preexistente

-Alfabetización informativa: capacidad para evaluar la calidad y validez de la información

-Alfabetización diversificada: capacidad para construir conocimiento en un entorno de navegación no lineal o hipertextual

-Alfabetización socioemocional: capacidad para comprender las reglas que impone el ciberespacio, y comprender su aplicación en procesos de comunicación online

Estas investigaciones y análisis han venido a mostrar la importancia de disponer de un conocimiento básico previo que haga posible la contrastación de las informaciones a que se accede, la capacidad de asignación de significados, la capacidad para relacionar las informaciones y la capacidad de valorar el grado de validez o relevancia de las mismas en la construcción de conocimiento y resolución de problemas. Y es en este cometido en el que se inicia la aparición de una nueva brecha entre los ciudadanos que han podido acceder a la adquisición y desarrollo de las competencias necesarias para la contrastación y asignación de significados y aquellos que, desde una pasividad inducida por sus desconocimientos previos, operan en el entorno digital como usuarios pasivos.

Para van Dijk y Hacker (van Dijk y Hacker, 2003), la resolución del problema de la llamada 'brecha digital' supone más que la superación de las desigualdades vinculadas a la capacidad adquisitiva, la posibilidad de conexión, la edad, la ocupación o el sexo (que han sido las variables tradicionalmente consideradas en los estudios empíricos). Los estudios que han considerado estas variables están relacionados con el uso del hardware y software, lo cual puede resolverse a medida que los usuarios desarrollen habilidades operativas; no obstante no se ha abordado sistemáticamente el análisis de las diferencias relacionadas con el 'para qué' se usan estas habilidades, ni qué habilidades se necesitan para buscar, seleccionar y utilizar la información que se puede obtener utilizando las múltiples plataformas de información. Desde nuestro punto de vista, todo ello está relacionado con el dominio de conocimientos y competencias previas que en gran medida no son exclusivamente deudoras del entorno digital. "Cuando las generaciones mayores han aprendido habilidades digitales operacionales y formales pueden actuar tan bien $\mathrm{o}$ incluso mejor que las generaciones jóvenes en entornos dado el conocimiento y saber que han desarrollado a lo largo de su vida" (van Dijk y van Deursen, 2010, 15). ${ }^{17}$

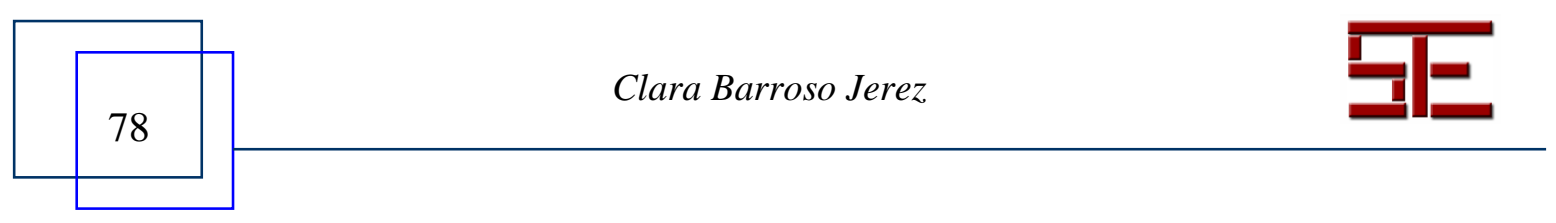




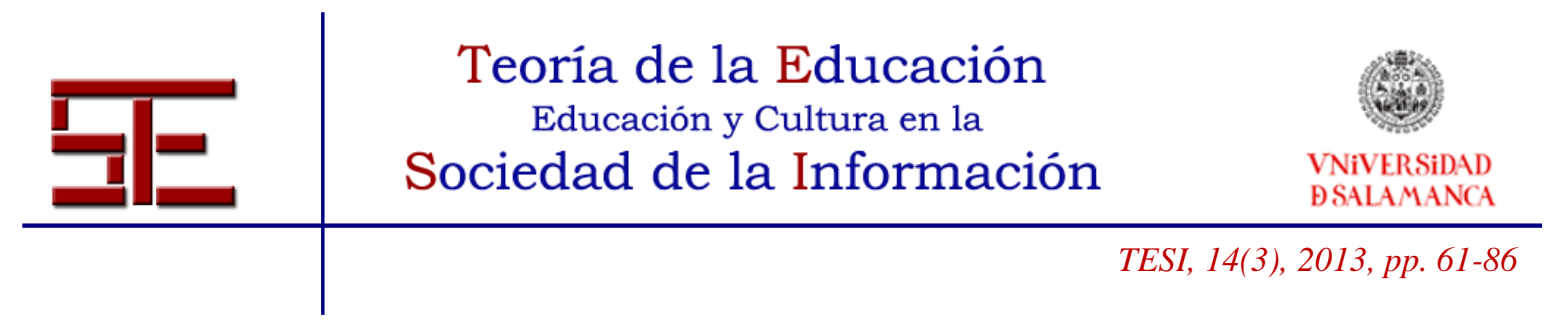

Estas investigaciones vienen a mostrar carencias digitales vinculadas al dominio de conocimientos previos, lo que dificulta la capacidad para utilizar los beneficios que aporta el entorno digital en el ámbito de los desempeños profesionales, en el ámbito de los aprendizajes escolares, o en la capacidad para ser un miembro activo de la sociedad de la información mediante el vínculo y participación con su comunidad.

\begin{abstract}
En una sociedad de la información, la información se conoce como lo que llamamos un bien primario. Todo el mundo lo necesita para funcionar en sociedad. Sin embargo, las personas también necesitan un capital cultural y social para usar la información de modo adecuado - esto es, las habilidades para seleccionar y procesar información y ser capaces de usarla en situación social y en red [...] En la actualidad varias tendencias se aúnan para promover desigualdad social de la información: la diferenciación social y cultural o individualización, el incremento en la diferencia de ingresos, la privatización y recortes en servicios públicos y sociales y, finalmente, el incremento de tecnología digital multifuncional y los diferentes modos de uso. La última tendencias apoya la visión de Van Dijk de los nuevos medios de comunicación como amplificadores de igualdades y desigualdades: Las igualdades y desigualdades ya presentes, creciendo o disminuyendo en la sociedad, se verán reforzadas por esta tecnología (van Dijk y Hacker, 2003, 324). ${ }^{18}$
\end{abstract}

Los estudios llevados a cabo por van Dijk y van Deursen muestran que, si bien las habilidades operativas y formales son necesarias en el entorno digital, no son suficientes. Sus resultados señalan que el nivel educativo y la experiencia juegan un importante papel en la capacidad para utilizar los recursos de dicho entorno. Es de destacar el énfasis puesto en las diferentes estrategias necesarias para aprender y desarrollar las habilidades necesarias en el entorno digital. Desde su perspectiva, las habilidades operacionales y formales pueden adquirirse básicamente mediante la práctica; no es así cuando se abordan las habilidades de tipo estratégico que serán deudoras de competencias más vinculadas al dominio de conocimientos. Esto origina su propuesta de una revisión del uso del entorno digital que se lleva a cabo en el desarrollo curricular.

En consecuencia, el potencial del entorno digital de la sociedad de la información en los procesos de construcción de conocimiento, es relativa a las competencias previas de los individuos. Las posibilidades que amplifica el entorno digital y los recursos cognoscitivos que este entorno supone son cualitativamente diferentes entre individuos con dominio de conocimientos previo, y los individuos que se sitúan ante este entorno esperando un suministro de información ante el que carecen de recursos para dar significado a la información que obtienen. Dicho de otro modo, las lindes de esta brecha digital están localizadas en el impulso y consumo masivo de información, que reduce la percepción de los límites y capacidades del entorno digital provocando un conocimiento limitado, frente a la de los individuos con competencias intelectuales para comprender el entorno digital, sus potencialidades y limitaciones, y capacitados para dar significado a la información y reconocerse como individuos activos en la construcción de la sociedad del conocimiento.

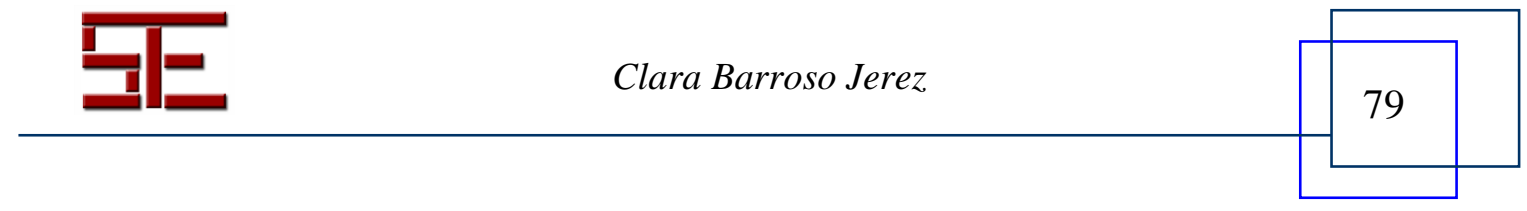




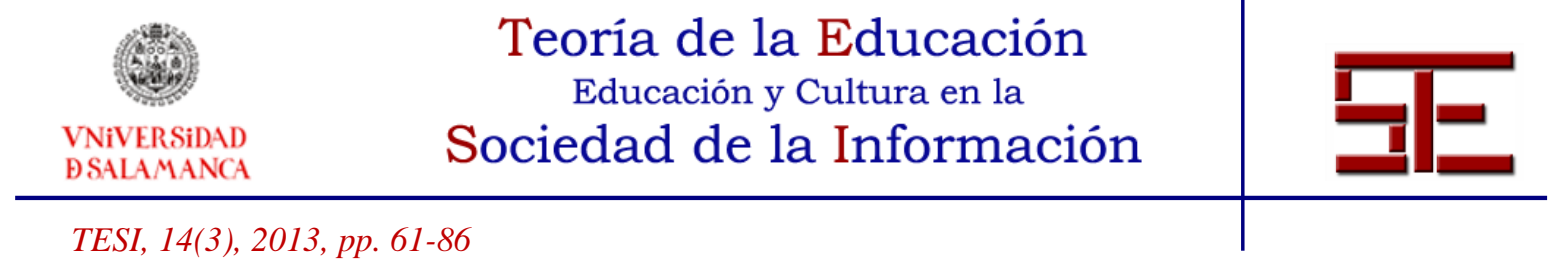

Se debería recapacitar sobre los compromisos pedagógicos que se han de asumir para superar esta "brecha digital". Las investigaciones orientadas al conocimiento de los procesos involucrados en la construcción de significados, el reconocimiento de la multitud de informaciones que los humanos utilizan para dar significado a cada información, de acuerdo con la percepción del contexto en que dicha información se produce y es utilizada, parecen relevantes para comprender los principios sobre los que se fundamenta el desarrollo de un sistema de conocimiento humano. La educación, como instrumento que permite compartir conocimiento, debe traspasar los estrechos límites de la información o formación para abarcar la adquisición y desarrollo de las facultades humanas necesarias para comprender, valorar y capacitar en el proceso de desarrollo del conocimiento.

Parafraseando a J. Dewey en su obra Democracia y Educación (Dewey, 2004): "Toda sociedad está compuesta por los individuos que reconocen los objetivos de la misma y se reconocen como parte integrante de los procesos necesarios para alcanzar estos objetivos. Cada individuo recoge la experiencia de su sociedad y la enriquece con su propia experiencia, participando, de este modo, en el desarrollo de su sociedad". En la sociedad del conocimiento cada individuo ha de poder reconocer y aceptar el objetivo de desarrollo del conocimiento; ha de saberse reconocer como parte integrante para el logro de dicha sociedad; y, por último, ha de ser capaz de recoger el conocimiento socialmente válido aportando su propio conocimiento para participar en el desarrollo del mismo. El entorno digital puede constituir, en este proceso, un instrumento muy valioso siempre que suponga un incremento del logro de dicho objetivo, no un límite al desarrollo individual y social, al desarrollo humano.

\section{CONCLUSIÓN}

Como se ha señalado, la difusión social de los instrumentos en que se funda la existencia de una sociedad de la información (abaratando costes, mejorando los procesos de interacción usuario-hardware, usuario-software) no garantiza la constitución de una sociedad del conocimiento. La sociedad del conocimiento, que se fundamente en la utilización del entorno digital, requiere de capacidades individuales que hagan posible que la utilización del entorno digital permita a los usuarios no sólo operar en dicho entorno como sujetos pasivos sino, más allá, utilizar dicho entorno para construir conocimiento utilizando competencias intelectuales que les han de permitir asignar significado a las informaciones a las que acceden. Estas capacidades están fuertemente vinculadas al dominio previo de habilidades, competencias y conocimientos de procesos de análisis y utilización significativa de las informaciones a que se accede mediante el entorno digital.

Podemos señalar dos ámbitos vinculados a este cometido. Por un lado, las capacidades o habilidades meramente operativas vinculadas a la utilización de herramientas básicas

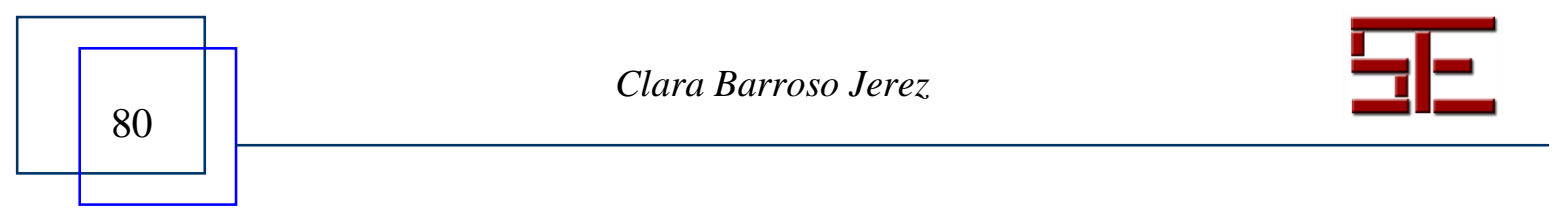




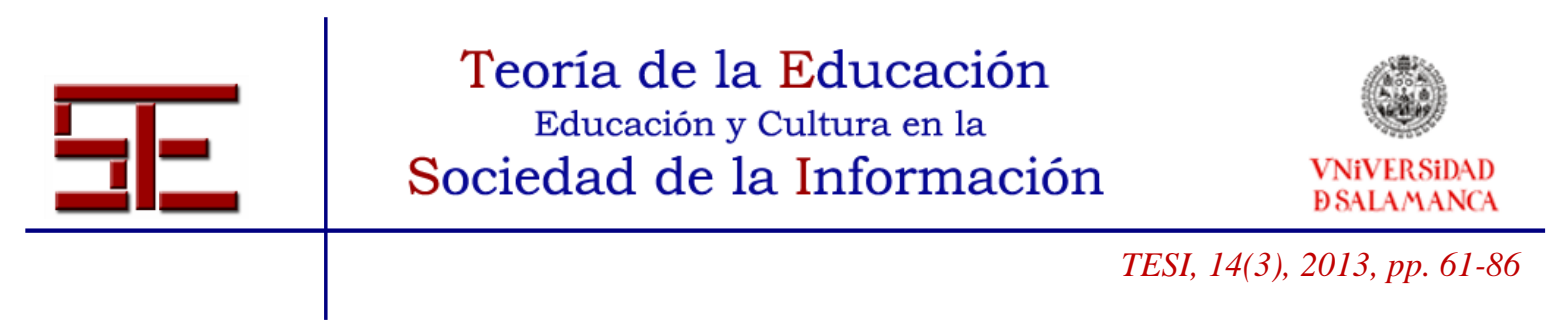

del entorno. Por otro las capacidades intelectuales, o dicho de otro modo las competencias, necesarias para utilizar el conocimiento previo como instrumento de búsqueda y valoración de las informaciones a que se puede acceder. En este cometido son básicos el dominio de razonamiento y el dominio de la construcción de un pensamiento crítico que faculte a los individuos para saber utilizar los instrumentos y herramientas del entorno digital en su proceso de construcción y desambiguación de la información a que accede en este entorno y dotarla de significado. Este proceso podría impulsar el entorno digital como instrumento de desarrollo humano y, en consecuencia, apoyaría a los individuos en su proceso de participación activa en la construcción de una sociedad del conocimiento.

En el entorno que constituye nuestra sociedad, la difusión masiva de los instrumentos y las herramientas informáticas ha desembocado en un proceso generalizado de captura de información. Estamos informados, casi instantáneamente, de todo aquello que parece relevante al devenir de nuestra vida social, si bien estas informaciones no siempre se fundamentan en la validez o la realidad de los ítems que componen la información que recibimos, ni tampoco queda garantizada su relevancia para el análisis y/o solución de los problemas que sí son subjetiva o colectivamente relevantes. Si bien se han incrementado las posibilidades y habilidades básicas para utilizar instrumentos y herramientas propios del entorno digital, no parece que el ritmo a que se han desarrollado éstas sea el mismo que el que han seguido el desarrollo de las capacidades y competencias necesarias para comprender, valorar y evaluar las informaciones, es decir aquellas competencias que permitan construir conocimiento a partir de las informaciones que recibimos masivamente a través de los diferentes canales que constituyen la sociedad de la información.

Esta situación se agrava a medida que se agranda la distancia entre la capacidad de acceso a la información, y las competencias cognitivas e intelectuales y el dominio de conocimientos previos que el individuo posee. Cuando las posibilidades de acceder a la información se incrementan, mientras las competencias vinculadas al dominio del conocimiento no se incrementan, la posibilidad de que la información sea un medio de manipulación social se amplifica, y eso es hoy posible como en ningún otro momento de la historia de la humanidad.

En esta dinámica podemos responsabilizar a las industrias, como impulsoras del consumo generalizado de los diversos canales de información, pero también podemos localizar las responsabilidades de los sistemas educativos que, si bien se han sumado a la dinámica del consumo de estos medios de información, han sido incapaces de estimular las competencias necesarias para construir conocimiento. Las prácticas didácticas, fuertemente deudoras del logro de objetivos de aprendizaje de contenidos, no han sabido fundamentar el aprendizaje sobre el valor del conocimiento y el valor del desarrollo de

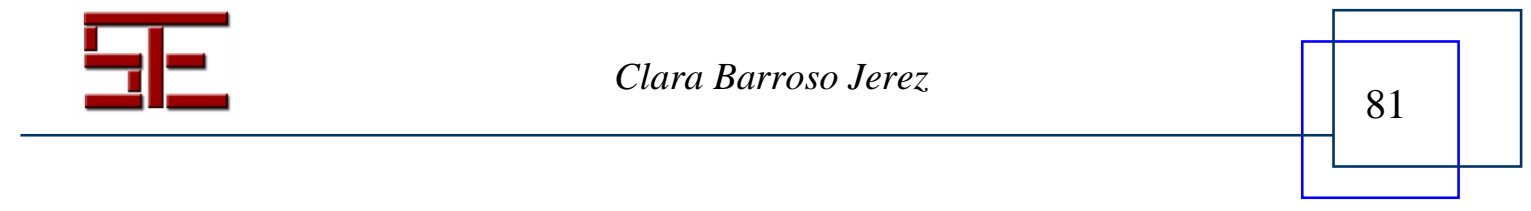




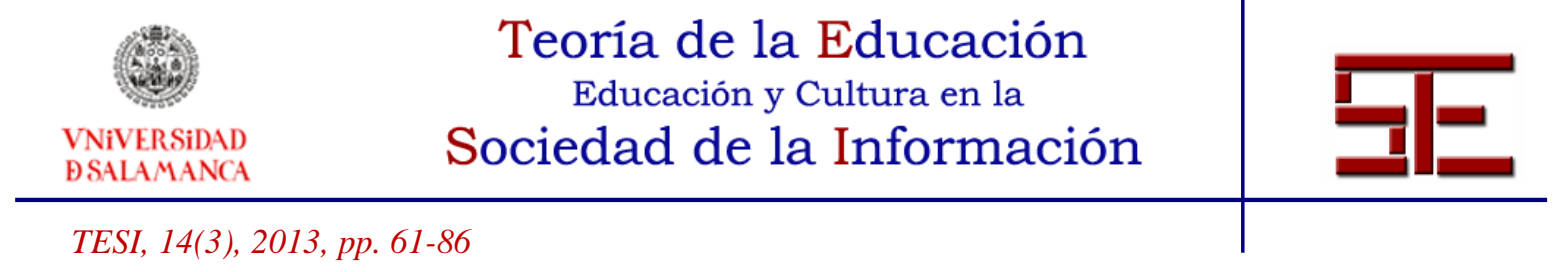

competencias de análisis racional y contrastación de informaciones para el desarrollo de conocimientos válidos.

En la actualidad impulsar los procesos de democratización del acceso a la información, mediante la generalización del uso del los entorno digital, debe vincularse al aprendizaje y desarrollo de competencias intelectuales relacionadas con el desarrollo de pensamiento y estrategias racionales que hagan posible la contrastación de las informaciones, la comprensión de los métodos y procesos utilizados para obtener la información y las facultades necesarias para asignar significados válidos a las mismas. Olvidar estas competencias puede incrementar el riesgo de convertir al entorno digital en el instrumento que aumente la brecha entre los individuos preparados para utilizarlo en la construcción del conocimiento, y los individuos que se convierten en usuarios pasivos y con pensamiento secuestrado por el mismo.

Ante el riesgo de ahondar en una nueva brecha social, la educación ha de reaccionar para actuar con objetivos que superen los meros intereses de la industria, básicamente interesada en incrementar el número de usuarios. La educación deberá potenciar la adquisición de las habilidades formales y estratégicas, y las competencias asociadas a la adquisición y desarrollo del dominio de conocimientos, que sitúe a los individuos en disposición de ser capaces de conocer y comprender el entorno digital y desarrollar la comprensión de los procesos que hacen posible la construcción de conocimiento válido, la construcción activa de conocimiento, así como el valor del conocimiento compartido.

El conocimiento compartido ha hecho evolucionar a la especie humana; hoy, más que nunca, es necesario recuperar el valor de ese conocimiento compartido como motor de progreso. Recuperar el valor de los procesos, sociales e individuales, que hagan posible el logro de objetivos vinculados al desarrollo humano más allá del desarrollo de la sociedad de la información. Ello no será posible sin detectar y superar cuáles son las brechas que en la actualidad dividen a la sociedad de la información en individuos capacitados para ser activos en la construcción de la sociedad del conocimiento y los individuos pasivos, meros usuarios y consumidores, de los artefactos que conforman el entorno digital.

\section{REFERENCIAS}

Bonfadelli, H. (2002): The Internet and Knowledge Gaps. A Theoretical and Empirical Investigation, European Journal of Communication, 17, 65-84

Bruner, J. (2001): El proceso mental en el aprendizaje. Madrid, Narcea.

Commoner, B. (1992): En paz con el Planeta. Barcelona, Crítica.

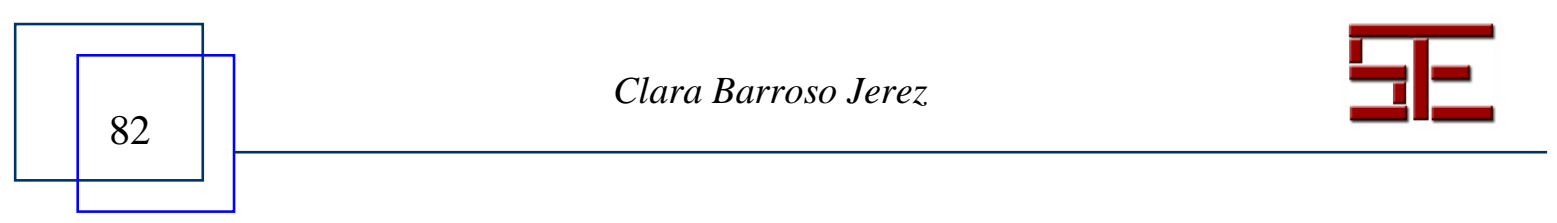




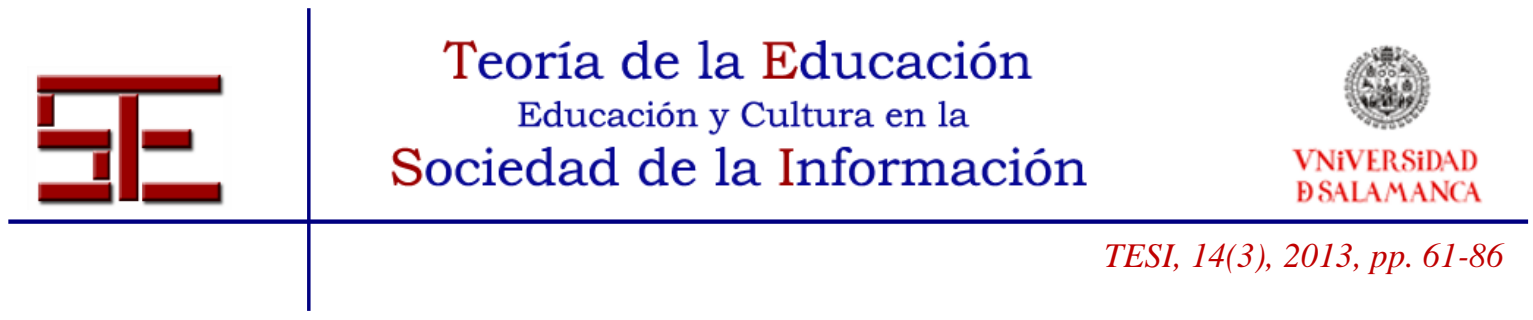

Dewey, J. (1910): How We Think . Boston, New York, Chicago, D.C.Heath \& Co. Pub

Dewey, J. (2004): Democracia y educación. Madrid, Morata.

Dewey, J. (2007): Cómo pensamos. Barcelona, Paidós

Deursen, A.J.A.M. van y Dijk, J.A.G.M. van (2009): Improving digital skills for the use of online public information and services. Elsevier, Government Information Quarterly, 26, 333-340

Deursen, A.J.A.M. van y Dijk, J.A.G.M. van (2009): Using the Internet: Skill related problems in users' online behavior. Interacting with Computers, doi:10.1016/j.intcom.2009.06.005

Deursen, A.J.A.M. van y Dijk, J.A.G.M. van (2010): 'Measuring Internet Skills', International Journal of Human-Computer Interaction, 26, 891 - 916

Dijk van J.A.G.M. y Hacker, K (2003): The Digital Divide as a Complex and Dynamic Phenomenon. The information Society, 19, 315-326

Dijk, J.A.G.M. van y Deursen, A. van (2010): Traditional Media Skills And Digitalmedia Skills: Much Of A Difference? Ponencia presentada en: International Communication Association Conference, Singapore, Republic of Singapore.

Eshet-Alkalai, y Amichai-Hamburger (2004): Experiments in Digital Literacy. Cyberpsychology \& Behavior, 7, 421-429

Gates, B.; Myhrvold, N. y Rinearson, P. (1996): The Road Ahead. New York, Viking Penguin.

Marchionini, G. y White, R. (2010): Find What You Need, Understand What You Find. International Journal of Human-Computer Interaction, 23:3 205-237

Popper, K.R. (1962): La lógica de la investigación científica. Madrid, Tecnos

Trillas, E. (1998): La inteligencia artificial. Máquinas y personas. Madrid, Debate

Ministerio de Industria y Energía de España (2012): Indicadores de seguimiento de la sociedad de la información. en: http://www.ontsi.red.es/ontsi/es/estudios-informes

Mora, F. (2002): Cómo funciona el cerebro. Madrid, Alianza

Potter, W.J. (2004): Theory of Media Literacy. A Cognitive Approach. Londres, Sage Publications, Inc.

\section{NOTAS}

1 En lo sucesivo se utilizarán los conceptos de "evento" y "acontecimiento" de acuerdo con la definición de Popper (Popper, 1962).) Un acontecimiento es descrito por un enunciado universal; un evento des-

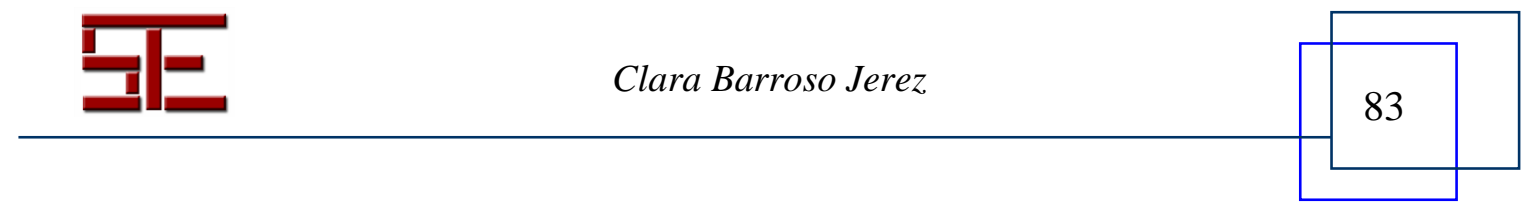




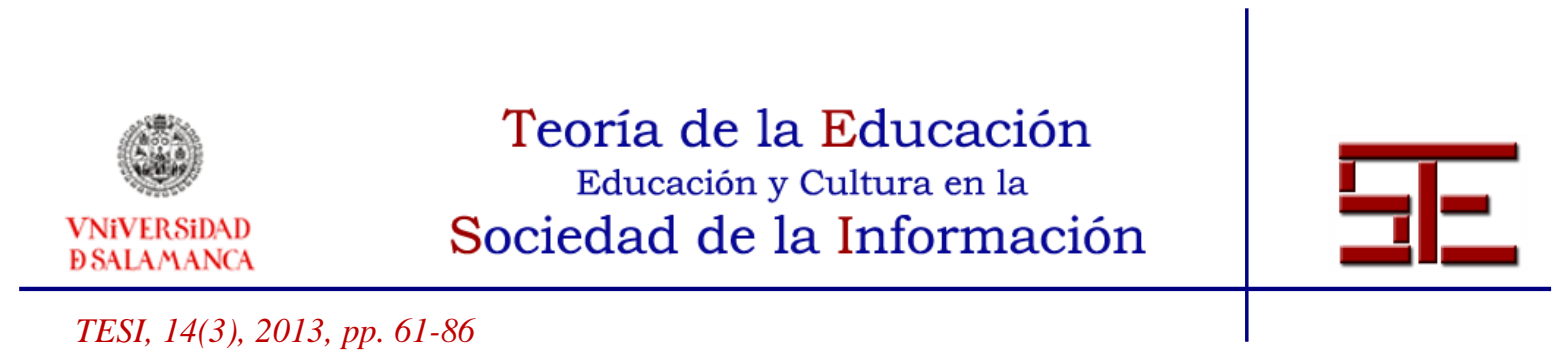

cribe un acontecimiento dentro de una región espacio-temporal determinada y se corresponde con un enunciado particular. En consecuencia, los eventos pueden formar parte del acervo de experiencias perceptivas; los acontecimientos son representaciones cognitivas que describen características universales de conjuntos de eventos.

2 "La adquisición de definición y de consistencia de significados deriva ante todo de la actividades prácticas [...] En la medida en que nos situamos en actitud pasiva ante los objetos, éstos no se distinguen de la vaga nebulosa que los rodea a todos" (Dewey, 2007, 151).

3 'Symbols are themselves, as pointed out above, particular, physical, sensible existences, like any other things. They are symbols only by virtue of what they suggest and represent, i.e. meanings [...] They stand for these meanings to any individual only when he has had experience of some situation to which these meanings are actually relevant. Words can detach and preserve a meaning only when the meaning has been first involved in our own direct intercourse with things" (Dewey, 1910, 176).

4 "If a person comes suddenly into your room and calls out "Paper," various alternatives are possible. If you do not understand the English language, there is simply a noise which may or may not act as a physical stimulus and irritant. But the noise is not an intellectual object; it does not have intellectual value. To say that you do not understand it and that it has no meaning are equivalents. If the cry is the usual accompaniment of the delivery of the morning paper, the sound will have meaning, intellectual content; you will understand it. Or if you are eagerly awaiting the receipt of some important document, you may assume that the cry means an announcement of its arrival. If (in the third place) you understand the English language, but no context suggests itself from your habits and expectations, the word has meaning, but not the whole event" (Dewey, 1910, 117).

5 Véase Christiansen, H. y Dahl, V. (2005) Meaning in Context. En Dey, A.K. et al. (Eds.) CONTEXT 2005. Lecture Notes in Computer Science, 3554, 97-111. Heidelberg, Springer-Verlag

6 "All knowledge, all science, thus aims to grasp the meaning of objects and events, and this process always consists in taking them out of their apparent brute isolation as events, and finding them to be parts of some larger whole suggested by them, which, in turn, accounts for, explains, interprets them; i.e. renders them significant" (Dewey, 1910, 117).

7 Se utiliza la distinción entre certeza y certidumbre utilizada en Lógica. La certeza hace referencia a la verdad o validez de una proposición; la certidumbre hace referencia a la probabilidad de verdad o validez.

8 "We reflect in order that we may get hold of the full and adequate significance of what happens. Nevertheless, something must be already understood, the mind must be in possession of some meaning which it has mastered, or else thinking is impossible" (Dewey, 1910, 119).

9 Los datos disponibles indican que en $200436 \%$ de los europeos usaban regularmente Internet, En España el valor era del 31\%. En 2010 los valores alcanzados eran, respectivamente, el $65 \%$ y el $58 \%$ (fuente EUROSTAT). Ministerio de Industria y Energía de España, 2012.

10 "Para abordar la representación del razonamiento ordinario es indispensable poder gestionar su imprecisión de manera flexible; a eso ayuda la lógica borrosa y a ello se deben los éxitos que, con ella, se obtienen con sistemas describibles por medio de reglas a las que asignar un factor de certeza que unas veces es numérico y otras lingüístico" (Trillas, 1998, 131).

11 "Demand for the solution of a perplexity is the steadying and guiding factor in the entire process of reflection. Where there is no question of a problem to be solved or a difficulty to be surmounted, the

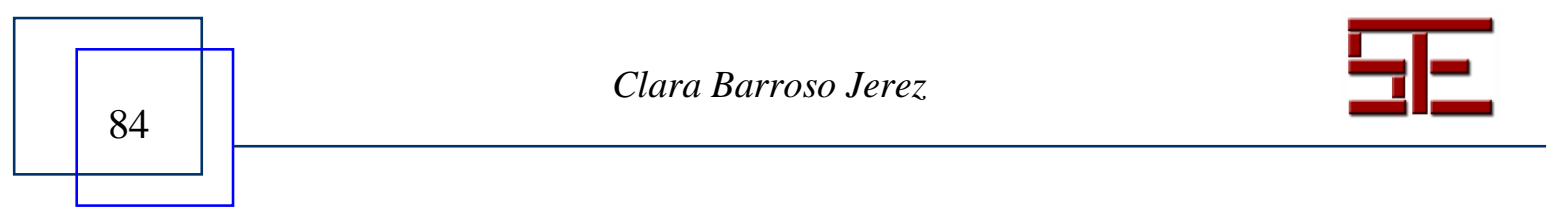




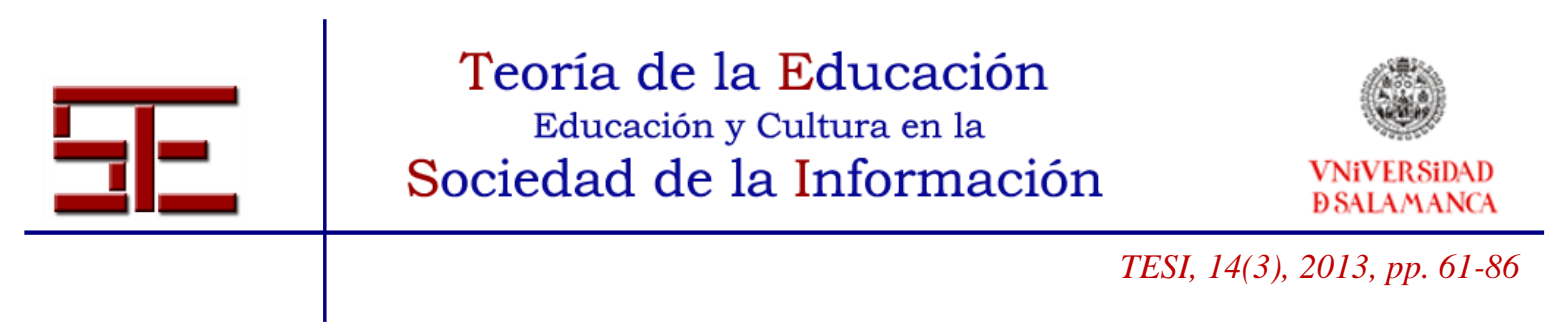

course of suggestions flows on at random" (Dewey, 1910, 29).

12 "These systems typically make many assumptions about the search environment in which they operate and the searchers that use them" (Marchionini y White, 2010, 227).

13 "Search systems typically allow Boolean expressions and offer advanced search operators such as quotation marks that can improve the precision of search results but must be learned and included in query statements. However, most users are unaware of these operators, mainly because their use is not publicized and the interface to compose queries with them is hidden from initial view. As a result, most users lack the additional skills required to formulate well-defined query statements" (Marchionini y White 2010, 211)

14 "Because the quality of queries directly affects the quality of search results, considerable attention has been paid to eliciting complete and accurate problem descriptions from information seekers. Query formulation requires two types of mappings: a semantic mapping of the information seeker's vocabulary used to articulate the task onto the system's vocabulary used to gain access to the content, and an action mapping of the strategies and tactics that the information seeker deems best to advance the task to the rules and features that the system interface allows. The search queries that emerge from query formulation are only an approximate, or "compromised" information need and may fall short of the description necessary to retrieve relevant documents simply because the vocabularies of the seeker and the system differ too greatly" (Marchionini y White, 2010: 210).

15 "Information seeking is taken to be a human activity that is part of some larger life activity. It might take place in a few seconds or over a lifetime, may be highly discrete, or may be integrated into the rhythms of daily life. Colloquially, information seeking and search are synonymous; however, we make the distinction that information seeking is a uniquely human activity and search can be undertaken by both machines and humans. [...] Although information seeking is driven by human needs and behaviors and thus highly variable, there are several common subactivities that may be supported by good technical design. Ultimately, well-designed search systems aim to support these subactivities and the overall information-seeking process. At present, most search systems focus on one or a few of these subactivities. As long as they are compatible with other kinds of information-processing applications that support the larger goals that motivate search, this is adequate, although we look for more comprehensive systems in the future" (Marchionini y White 2010, 207).

${ }_{16}$ "Media literacy is the set of perspectives from which we expose ourselves to the media and interpret the meaning of the messages we encounter. We build our perspectives from knowledge structures. The knowledge structures form the platforms on which we stand to view the multifaceted phenomenon of the media: their business, their content, and their effects on individuals and institutions [...] The more people use these knowledge structures in mindful exposures, the more they will be able to use media exposures to meet their own goals and the more they will be able to avoid high risks for negative effects" (Potter, 2004, 58-59).

17 "When the elderly generations have learned operational and formal digital skills they can perform just as well, or even better in digital environments - given the knowledge and wisdom they have developed during their life- than the younger generations" (van Dijk y van Deursen, 2010, 15).

${ }_{18}$ "In an information society, information is known as a so called primary good. Everybody needs it to function in society. However, people also need cultural capital and social capital to use information in appropriate ways - that is, the skills to select and process information and be able to use it in one's social position and network. [...] In the present epoch several trends come together to promote information inequality: social and cultural differentiation or individualization, rising income differentials,

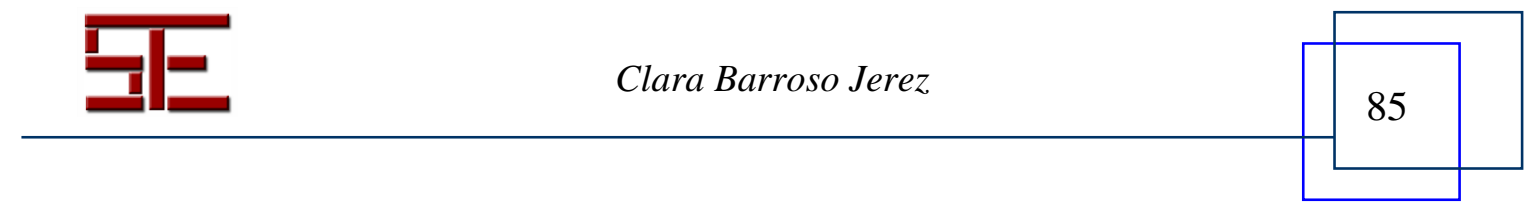




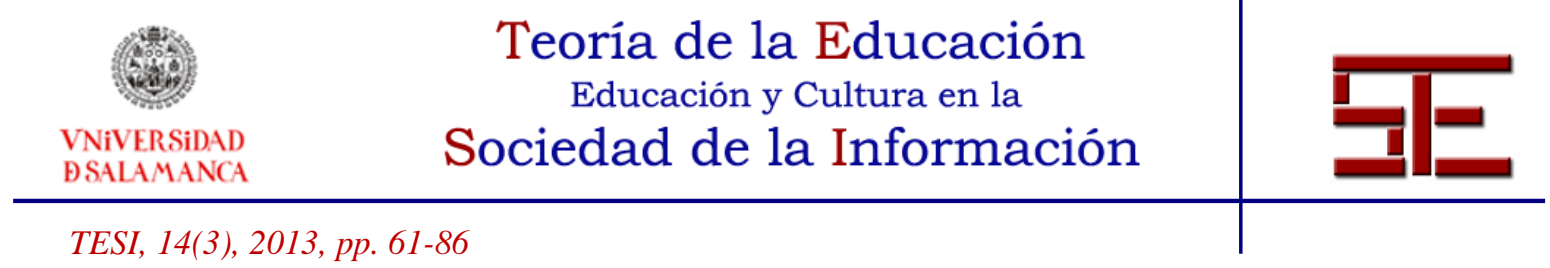

privatization and cutbacks in social and public services, and, finally, multifunctional and differently used digital technology. The last trend supports Van Dijk's vision of the new media as trend amplifiers: Equalities and inequalities already present, growing or declining in society will be reinforced by this technology" (van Dijk y Hacker, 2003, 324).

Para citar el presente artículo puede utilizar la siguiente referencia:

Barroso Jerez, C. (2013). Sociedad del conocimiento y entorno digital. Revista Teoría de la Educación: Educación y Cultura en la Sociedad de la Información. 14(3), 61-86 [Fecha de consulta: dd/mm/aaaa].

http://campus.usal.es/ revistas_trabajo/index.php/revistatesi/article/view/11351/11783

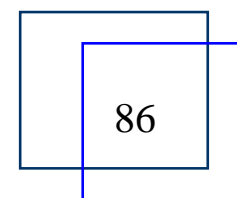

